\title{
Foundation of Interval-Valued Intuitionistic Fuzzy Limit and Differential Theory and an Application to Continuous Data
}

\author{
Zhenghai Ai, ${ }^{1}$ Xiaoqin Shu, ${ }^{2}$ and Zeshui $X u \mathbb{D}^{3}$ \\ ${ }^{1}$ College of Mathematics and Information Science, Leshan Normal University, Leshan, \\ Sichuan 614000, China \\ ${ }^{2}$ School of Physics and Electronic Engineering, Leshan Normal University, Leshan, \\ Sichuan 614000, China \\ ${ }^{3}$ State Key Laboratory of Hydraulics and Mountain River Engineering, Sichuan University, \\ Chengdu 610064, China
}

Correspondence should be addressed to Zeshui Xu; xuzeshui@263.net

Received 27 July 2019; Accepted 9 September 2019; Published 27 October 2019

Academic Editor: Toshikazu Kuniya

Copyright (c) 2019 Zhenghai Ai et al. This is an open access article distributed under the Creative Commons Attribution License, which permits unrestricted use, distribution, and reproduction in any medium, provided the original work is properly cited.

\begin{abstract}
The intuitionistic fuzzy calculus (IFC), based on the basic operational laws of intuitionistic fuzzy numbers (IFNs), has been put forward. However, the interval-valued IFC (IVIFC), based on the basic operational laws of interval-valued IFNs (IVIFNs), is only in the original stage. To further develop the theory of the IVIFC and make it be rigorous, the primary task is to systematically investigate the characteristics of the limits and differentials, which is a foundation of the IVIFC. Moreover, there is quite a lot of literature on IVIFNs; however, the scholars did not reveal the relationships between IFNs and the IVIFNs. To do that, we first investigate the limit of interval-valued intuitionistic fuzzy sequences, and then, we focus on investigating the limit, the continuity, and the differential of IVIFFs in detail and reveal their relationships. After that, due to the fact that the IFC and the IVIFC are based on the basic operational laws of IFNs and IVIFNs, respectively, we reveal the relationships between the IFNs and the IVIFNs via some homomorphic mappings. Finally, a case study about continuous data of IVIFNs is provided to illustrate the advantages of continuous data.
\end{abstract}

\section{Introduction}

Since Zadeh [1] introduced fuzzy sets (FSs) in 1965, a lot of theories addressing vagueness and uncertainties of the data have been proposed [2-6]. The intuitionistic fuzzy set (IFS) [2] is one of the most widely used extensions of the FSs. Compared with the FS, the IFS is an effective approach to describe vagueness and uncertainties by adding a nonmembership degree to each element. Later on, Atanassov and Gargov [7] presented the definition of interval-valued intuitionistic fuzzy set (IVIFS), in which the membership degree and the nonmembership degree are two interval values instead of two crisp numbers. To some extent, interval values can better reveal the characteristics of things: vagueness and uncertainties. Due to the advantages of the IVIFS, it has been investigated widely and has been successfully applied to many fields, such as aggregation operators [8,9], decision-making $[10,11]$, preference relations $[12,13]$, entropy $[14,15]$, and so on.

More recently, the scholars have investigated a series of fundamental properties with respect to the intuitionistic fuzzy calculus (IFC) [16-22]; the key characteristic of the IFC is that it is based on the basic operational laws of intuitionistic fuzzy numbers (IFNs). With these operational laws, Lei and $\mathrm{Xu}$ [16] introduced the limits, the 
derivatives, and the differentials in intuitionistic fuzzy circumstance and gave their basic properties. To further develop limit theory, the intuitionistic fuzzy infinitesimals (IFIs) [17] were introduced to reveal the relationships between the derivatives and the differentials. Later on, the completeness of intuitionistic fuzzy partially ordered set [18] was discussed. As for the definite integrals in the IFC, the scholars constructed several kinds of definite integrals [19-22] from different points of view. Nevertheless, interval-valued IFC (IVIFC), which is based on the operational laws of the interval-valued IFNs (IVIFNs), is only in the original stage. Zhao et al. [23] first presented four change directions of the IVIFNs and then introduced the limits, the derivatives, and the differentials in the intervalvalued intuitionistic fuzzy circumstance, based on which some basic properties were proposed.

In what follows, let us recall an interesting story: "Really extensive work on series began about 1730 with Euler aroused tremendous interest in the subject. But there was much confusion in his thinking. Euler argued that since $1 /(1-x)=1+x+x^{2}+\cdots+x^{n}+\cdots$ then $1 / 2=1-1+1-$ $1+1 \cdots$ as $x=-1$, so that the sum is $1 / 2$ " [24]. Even though Euler is such a very famous mathematician he also made a mistake which seems very obvious for modern mathematical workers. The reason why Euler made an obvious mistake is that the limit theory was not very rigorous at that time, so "there was much confusion in his thinking." From the story, we can see that the limit theory plays a fundamental and significant role in the traditional calculus. Meanwhile, the importance of the differential is self-evident, which is the base of the definite integral and the indefinite integral. As aforementioned, the IVIFC is only in its original stage, and thus, to further develop the theory of the IVIFC and make it be a rigorous subject, the primary task is to systematically investigate the characteristics of the limits and the differentials, which are the foundation of the IVIFC.

As is known to all, the forms of the IVIFNs are more complicated than those of IFNs, which leads to that when we discuss the IVIFC, correspondingly, the forms in the IVIFC are more complicated than those in the IFC. In our opinion, it is an interesting issue and also a challenge that how to simplify the forms in the IVIFC. On the other hand, if we follow the thoughts in the IFC, we can easily obtain some corresponding results in the IVIFC. However, if we do so, it is no doubt that the computation is considerably tedious, and the IVIFC is only a counterpart of the IFC, the beauty of mathematics is not complicated computation but her brilliant thoughts [18]. Therefore, we do not prepare to follow the thoughts in the IFC and shall reduce some tedious computations as possible as we can. Finally, it might be well to point out that the IFN is a particular form of the IVIFN, which means that when we develop the theory of the IVIFC, we also improve the corresponding theory of the IFC if we transform the IVIFNs into the IFNs. In brief, the IVIFC and the IFC can promote each other, and the IVIFC is not a counterpart of the IFC.

To do so, the rest of this paper can be organized as follows: In Section 2, we give some preparations for the whole work. Section 3 discusses the limit theories of interval-valued intuitionistic fuzzy sequences. In Sections 4 and 5 , we focus on investigating the limit, continuity, and differential theory of IVIFFs in detail. Section 6 employs an application of continuous data in decision-making to demonstrate the advantages of continuous data. We compare the operational laws of IFNs with those of IVIFNs and interpret their relationships by some homomorphic mappings in Section 7. Finally, we offer some concluding remarks in Section 8.

\section{Preliminaries}

To begin with, let us recall the concept of IVIFS and reveal the relationship between the IVIFS and the IFS.

As described in Introduction, Atanassov and Gargov [7] generalized the IFS [2] to the IVIFS. An IVIFS $\widetilde{A}$ on $X$ is a mapping $\widetilde{A}: X=\left\{\left(x, \mu_{\widetilde{A}}(x), \nu_{\widetilde{A}}(x)\right) \mid x \in X\right\}$, where $\mu_{\tilde{A}}(x)$ and $\nu_{\tilde{A}}(x)$ are two intervals in $[0,1]$ with sup $\mu_{\tilde{A}}(x)+\sup \tau_{\tilde{A}}(x) \leq 1$. Based on the IVIFS, Xu [25] called the ordered pair $\left(\mu_{\tilde{A}}(x), \nu_{\widetilde{A}}(x)\right)$ an interval-valued intuitionistic fuzzy number (IVIFN). Obviously, when $\sup \mu_{\tilde{A}}$ $(x)=\inf \mu_{\widetilde{A}}(x)$ and $\sup v_{\widetilde{A}}(x)=\inf \nu_{\widetilde{A}}(x)$ for any $x \in X$, the given IVIFS and IVIFN are reduced to the IFS and the IFN, respectively.

In order to obtain two kinds of aggregation techniques, $\mathrm{Xu}$ [25] introduced some basic operational laws of IVIFNs, and Zhao et al. [23] further developed them and gave the subtraction and division operational laws of IVIFNs, which can be listed below.

Definition 1 (see $[23,25])$. Let $\widetilde{\alpha}_{1}=\left(\left[a_{1}, b_{1}\right],\left[c_{1}, d_{1}\right]\right)$ and $\widetilde{\alpha}_{2}=\left(\left[a_{2}, b_{2}\right],\left[c_{2}, d_{2}\right]\right)$ be two IVIFNs. Then,

$$
\begin{aligned}
\widetilde{\alpha}_{1} \oplus \widetilde{\alpha}_{2} & =\left(\left[1-\left(1-a_{1}\right)\left(1-a_{2}\right), 1-\left(1-b_{1}\right)\left(1-b_{2}\right)\right],\left[c_{1} c_{2}, d_{1} d_{2}\right]\right), \\
\widetilde{\alpha}_{1} \otimes \widetilde{\alpha}_{2} & =\left(\left[a_{1} a_{2}, b_{1} b_{2}\right],\left[1-\left(1-c_{1}\right)\left(1-c_{2}\right), 1-\left(1-d_{1}\right)\left(1-d_{2}\right)\right]\right), \\
\lambda \widetilde{\alpha}_{1} & =\left(\left[1-\left(1-a_{1}\right)^{\lambda}, 1-\left(1-b_{1}\right)^{\lambda}\right],\left[c_{1}^{\lambda}, d_{1}^{\lambda}\right]\right), \quad \lambda>0, \\
\widetilde{\alpha}_{1}^{\lambda} & =\left(\left[a_{1}^{\lambda}, b_{1}^{\lambda}\right],\left[1-\left(1-c_{1}\right)^{\lambda}, 1-\left(1-d_{1}\right)^{\lambda}\right]\right), \quad \lambda>0,
\end{aligned}
$$




$$
\begin{aligned}
& \tilde{\alpha}_{1} \ominus \widetilde{\alpha}_{2}= \begin{cases}\left(\left[\frac{a_{1}-a_{2}}{1-a_{2}}, \frac{b_{1}-b_{2}}{1-b_{2}}\right],\left[\frac{c_{1}}{c_{2}}, \frac{d_{1}}{d_{2}}\right]\right), & a_{1} \geq a_{2}, b_{1} \geq b_{2}, c_{1} \leq c_{2}, \\
& d_{1} \leq d_{2}, c_{2}>0, d_{2}>0, \\
& c_{1}\left(1-a_{2}\right) \leq c_{2}\left(1-a_{1}\right), \\
(0,1), & d_{1}\left(1-b_{2}\right) \leq d_{2}\left(1-b_{1}\right), \\
& \text { otherwise, }\end{cases} \\
& \tilde{\alpha}_{1} \oslash \tilde{\alpha}_{2}= \begin{cases}\left(\left[\frac{a_{1}}{a_{2}}, \frac{b_{1}}{b_{2}}\right],\left[\frac{c_{1}-c_{2}}{1-c_{2}}, \frac{d_{1}-d_{2}}{1-d_{2}}\right]\right), & a_{1} \leq a_{2}, b_{1} \leq b_{2}, c_{1} \geq c_{2}, \\
& d_{1} \geq d_{2}, a_{2}>0, b_{2}>0, \\
& a_{1}\left(1-c_{2}\right) \leq a_{2}\left(1-b_{1}\right), \\
& b_{1}\left(1-d_{2}\right) \leq b_{2}\left(1-d_{1}\right), \\
& \text { otherwise. }\end{cases}
\end{aligned}
$$

After investigating the addition, subtraction, multiplication, and division operational laws, Zhao et al. [23] defined four regions for a given IVIFN; the four regions of $\widetilde{\alpha}_{0}$ can be shown in Figure 1, where $\widetilde{I}$ is a collection of all IVIFNs and $A_{\tilde{\alpha}_{0}}^{\diamond}=\left\{\widetilde{\alpha}_{0} \diamond \widetilde{\beta} \mid \beta \in \widetilde{I}, \diamond \in\{\oplus, \otimes, \ominus, \oslash\}\right\}$. Based on the four regions of $\tilde{\alpha}_{0}$, correspondingly, they defined four types of limits. For brevity, below we only offer the notion of the addition limit.

Definition 2 (see [23]). Let $\left\{\widetilde{\alpha}_{n}\right\}$ be an addition sequence of $\widetilde{\alpha}_{0}$, that is, $\widetilde{\alpha}_{n} \in A_{\widetilde{\alpha}_{0}}^{\oplus}$ for all but finitely many $n$. If for a given $\widetilde{\varepsilon}=\left(\left[a_{\tilde{\varepsilon}}, b_{\tilde{\varepsilon}}\right],\left[c_{\tilde{\varepsilon}}, d_{\tilde{\varepsilon}}\right]\right)>_{L}(0,1)$, there exists a positive integer $N$, such that $\widetilde{\alpha}_{n} \ominus \widetilde{\alpha}_{0}<_{L} \widetilde{\varepsilon}$ for $n>N$, then $\widetilde{\alpha}_{0}$ is called the addition limit of $\left\{\widetilde{\alpha}_{n}\right\}$, and we write $\lim _{n \longrightarrow+\infty} \widetilde{\alpha}_{n}^{\oplus}=\widetilde{\alpha}_{0}$ or $\tilde{\alpha}_{n}^{\oplus} \longrightarrow \tilde{\alpha}_{0}$ as $n \longrightarrow \infty$. For $\tilde{\alpha}_{1}=\left(\left[a_{1}, b_{1}\right],\left[c_{1}, d_{1}\right]\right)$ and $\widetilde{\alpha}_{2}=\left(\left[a_{2}, b_{2}\right],\left[c_{2}, d_{2}\right]\right)$, the symbol $<_{L}$ indicates that $\widetilde{\alpha}_{1}<_{L} \widetilde{\alpha}_{2} \Longleftrightarrow \widetilde{\alpha}_{1} \leq_{L} \widetilde{\alpha}_{2}$ and $\widetilde{\alpha}_{1} \neq \widetilde{\alpha}_{2}$, and the symbol $\leq_{L}$ means $\widetilde{\alpha}_{1} \leq_{L} \widetilde{\alpha}_{2} \Longleftrightarrow a_{1} \leq a_{2}, b_{1} \leq b_{2}, c_{1} \geq c_{2}$ and $d_{1} \geq d_{2}$.

Besides, Zhao et al. [23] also introduced the concepts of continuous interval-valued intuitionistic fuzzy functions (IVIFFs) and investigated the properties with respect to differentiable IFFs; for the same reason, here we only list the concept and property concerning addition continuous and differentiable IVIFF.

Definition 3 (see [23]). Let $F(\widetilde{X})=\left(\left[f_{1}\left(\mu_{1}, \mu_{2}, v_{1}, v_{2}\right)\right.\right.$, $\left.\left.f_{2}\left(\mu_{1}, \mu_{2}, v_{1}, v_{2}\right)\right],\left[g_{1}\left(\mu_{1}, \mu_{2}, v_{1}, v_{2}\right), g_{2}\left(\mu_{1}, \mu_{2}, v_{1}, v_{2}\right)\right]\right)$ be an IVIFF of $\tilde{X}=\left(\left[\mu_{1}, \mu_{2}\right],\left[\nu_{1}, \nu_{2}\right]\right)$. If for any $\widetilde{\varepsilon}=\left(\left[\mu_{1 \tilde{\varepsilon}}, \mu_{2 \tilde{\varepsilon}}\right],\left[\nu_{1 \tilde{\varepsilon}}, v_{2 \tilde{\varepsilon}}\right]\right)>_{L}(0,1)$, there exists $\widetilde{\delta}=\left(\left[\mu_{1 \tilde{\delta}}\right.\right.$, $\left.\left.\mu_{2 \tilde{\delta}}\right],\left[\nu_{1 \tilde{\delta}}, v_{2 \tilde{\delta}}\right]\right)$, such that $\tilde{X} \in S^{\oplus}\left(\tilde{X}_{0}, F\right)=\left\{\tilde{X} \mid \tilde{X} \in A_{\widetilde{X}_{0}}^{\oplus}\right.$, $0 \leq\left(g_{i}\left(\mu_{1}, \mu_{2}, v_{1}, v_{2}\right) / g_{i}\left(\mu_{10}, \mu_{20}, v_{10}, v_{20}\right)\right) \leq\left(\left(1-f_{i}\left(\mu_{1}, \mu_{2}\right.\right.\right.$, $\left.\left.\left.\left.\nu_{1}, v_{2}\right) / 1\right)-\left(f_{i}\left(\mu_{10}, \mu_{20}, v_{10}, v_{20}\right)\right)\right) \leq 1, i=1,2\right\}$, we have $F(\tilde{X}) \ominus F\left(\tilde{X}_{0}\right)<_{L} \widetilde{\mathcal{\varepsilon}}$ for $(0,1)<{ }_{L} \tilde{X} \ominus \widetilde{X}_{0}<{ }_{L} \widetilde{\delta}$, then $F(\tilde{X})$ is additive continuous at $\widetilde{X}_{0}=\left(\left[\mu_{10}, \mu_{20}\right],\left[\nu_{10}, v_{20}\right]\right)$, denoted by $\lim _{\tilde{X}} \longrightarrow \tilde{X}^{\oplus} F(\tilde{X})=F\left(\widetilde{X}_{0}\right)$.

Incidentally, from Definition 3 we can see that both $F(\widetilde{X}) \ominus F\left(\widetilde{X}_{0}\right)$ and $\widetilde{X} \ominus \widetilde{X}_{0}$ are IVIFNs for any given $\widetilde{X} \in S^{\oplus}\left(\widetilde{X}_{0}, F\right)$. After giving the notion of continuity, it is natural to investigate the interval-valued intuitionistic fuzzy derivative and differential operations.

Theorem 1 (see [23]). Let $F(\tilde{X})=\left(\left[f_{1}\left(\mu_{1}, \mu_{2}, \nu_{1}, \nu_{2}\right), f_{2}\left(\mu_{1}\right.\right.\right.$, $\left.\left.\left.\mu_{2}, v_{1}, v_{2}\right)\right],\left[g_{1}\left(\mu_{1}, \mu_{2}, v_{1}, v_{2}\right), g_{2}\left(\mu_{1}, \mu_{2}, v_{1}, v_{2}\right)\right]\right)$ be an IVIFF

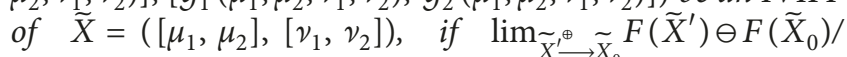
$\tilde{X}^{\prime} \ominus \widetilde{X}_{0}$ is an IVIFN, then we say that $F(\tilde{X})$ is addition differentiable at $\widetilde{X}_{0}=\left(\left[\mu_{10}, \mu_{20}\right],\left[\nu_{10}, v_{20}\right]\right)$. Moreover, if $F(\widetilde{X})$ is additive differentiable at $\widetilde{X}_{0}$, then $F(\tilde{X})=\left(\left[f_{1}\right.\right.$ $\left.\left.\left(\mu_{1}\right), f_{2}\left(\mu_{2}\right)\right],\left[g_{1}\left(\nu_{1}\right), g_{2}\left(\nu_{2}\right)\right]\right), 0 \leq\left(1-\mu_{20} / 1-f_{2}\left(\mu_{20}\right)\right) f_{2}^{\prime}$ $\left(\mu_{20}\right) \leq\left(\nu_{20} / g_{2}\left(\nu_{20}\right)\right) g_{2}^{\prime}\left(\nu_{20}\right) \leq 1,0 \leq\left(1-\mu_{10} / 1-f_{1}\left(\mu_{10}\right)\right) f_{1}^{\prime}$ $\left(\mu_{10}\right) \leq 1$, and $0 \leq\left(\nu_{10} / g_{1}\left(\nu_{10}\right)\right) \quad g_{1}^{\prime}\left(\nu_{10}\right) \leq 1$; in this case, we have $d F(\tilde{X}) /\left.d \widetilde{X}\right|_{\tilde{X}=\tilde{X}_{0}}=\left(\left[\left(1-\mu_{10} / 1-f_{1}\left(\mu_{10}\right)\right) f_{1}^{\prime}\left(\mu_{10}\right)\right.\right.$, $\left.\left(1-\mu_{20} / 1-f_{2}\left(\mu_{20}\right)\right) f_{2}^{\prime}\left(\mu_{20}\right)\right],\left[1-\left(\nu_{10} / g_{1}\left(\nu_{10}\right)\right) g_{1}^{\prime}\left(\nu_{10}\right), 1-\right.$ $\left.\left.\left(\nu_{20} / g_{2}\left(\nu_{20}\right)\right) g_{2}^{\prime}\left(\nu_{20}\right)\right]\right)$.

The order is $\leq_{L}$ in Definition 3. Indeed, the IFC has its own orders $\triangleleft$ and $\triangleleft_{\otimes}$, based on which we can define two kinds of neighborhoods of an IFN, and then we can obtain two kinds of accumulation points; here, we only list the first kind:

Definition 4 (see [18]). Let $(\alpha, \beta)=\{\gamma \mid \alpha \triangleleft \gamma \triangleleft \beta\}$ be an open interval, where $\alpha \triangleleft \beta \Longleftrightarrow \beta=\alpha \oplus \eta$ and $\eta \neq(0,1)$. We call $U\left(\alpha_{0}, \delta\right)=\left(\alpha_{0} \ominus \delta, \alpha_{0} \oplus \delta\right)$ a neighborhood of $\alpha_{0}$, if each $U\left(\alpha_{0}, \delta\right)$ contains infinitely many points of a set $S \subset I$, where $I$ is a collection of all IFNs, then $\alpha_{0}$ is called an accumulation point of the set $S$; if each $U\left(\alpha_{0}, \delta\right)$ only contains the point $\alpha_{0}$ in $S$, then $\alpha_{0}$ is called an isolated point of the set $S$.

Based on the above definition, Cantor intersection theorems can be obtained, whose roles are the same as Cantor intersection theorem in the classical calculus; here, we only list Cantor intersection theorem of the first kind.

Theorem 2 (see [18]). If $\left\{\left[\alpha_{n}, \beta_{n}\right]\right\}$ is a nested closed interval, then there exists an IFN $\gamma_{0}$ such that $\gamma_{0} \in\left[\alpha_{n}, \beta_{n}\right]$, $n=1,2, \cdots$, that is, $\alpha_{n} \unlhd \gamma_{0} \unlhd \beta_{n}$ for any $n \in N^{+}$, where $\gamma_{0}=$ $\lim _{n \longrightarrow \infty} \alpha_{n}=\lim _{n \longrightarrow \infty} \beta_{n}$. 


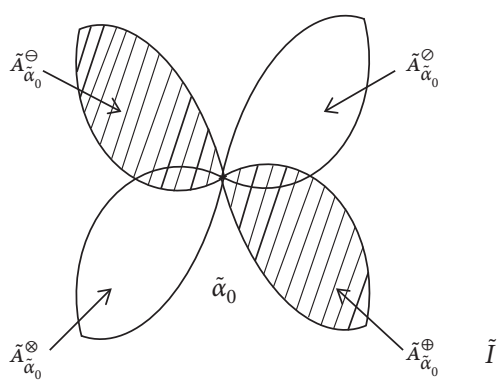

Figure 1: The four regions of $\widetilde{\alpha}_{0}$ [23].

As mentioned in Introduction, using the IFIs, we can reveal the relationships between the derivatives and the differentials, using the intuitionistic fuzzy infinitesimals and the operator $|\bullet|$ (if $\beta \unlhd \alpha$, then $|\alpha \ominus \beta|=\alpha \ominus \beta$, and if $\alpha \unlhd \beta$, then $|\alpha \ominus \beta|=\beta \ominus \alpha$.) [17]. The relationship between the derivatives and the differentials with respect to the addition and the subtraction can be obtained, and their relationships are the same as those of the derivative and the differential in the classical calculus: the relationship between the derivatives and the differentials with respect to the addition and the subtraction can be listed as follows.

Theorem 3 (see [17]). Let $F(X)=\left(f_{1}(\mu), f_{2}(v)\right)$ be an IFF of $X$, if $\lim _{X \longrightarrow X_{0}} F(X)=(0,1)$, then $F(X)$ is said to be an IFI at $X_{0}$. Let $F(X)$ and $G(X)$ be two IFIs at $X_{0}$, where $G(X)=\left(g_{1}(\mu), g_{2}(v)\right)$, if $F(X)=\left(o\left(g_{1}(\mu)\right), 1-o\left(1-g_{2}\right.\right.$ $(\nu))$ ), then we say that $F(X)$ is a higher order at $\widetilde{X}_{0}$ than $G(X)$, denoted by $F(X)=o(G(X)) . F(X)$ has the derivative at $X_{0}$, if and only if ||$F\left(X_{0}\right) \ominus F(X)\left|\ominus\left(A\left|\otimes X_{0} \ominus X\right|\right)\right|=$ $o\left(\left|X_{0} \ominus X\right|\right)$ for $X \in S^{\oplus}\left(X_{0}, F\right) \cup S^{\ominus}\left(X_{0}, F\right)$, where $A=F^{\prime}$ $\left(X_{0}\right)=\left(\left(1-\mu_{0} / 1-f_{1}\left(\mu_{0}\right)\right) f_{1}^{\prime}\left(\mu_{0}\right), 1-\left(v_{0} / f_{2}\left(\nu_{0}\right)\right) f_{2}^{\prime}\left(v_{0}\right)\right)$.

We end this section with the notions of homomorphism and isomorphism which emerge in abstract algebra.

Definition 5 (see [26]). Let $(A, \circ)$ and $(\bar{A}, \bar{\circ})$ be two algebraic structures. A homomorphism is a map from $A$ to $\bar{A}$ such that $\varphi(\alpha \circ \beta)=\varphi(\alpha) \circ \varphi(\beta)$ holds for all $\alpha, \beta \in A$, and if the map is a surjection, then we write $(A, \circ) \sim(\bar{A}, \bar{\circ})$ or $A \sim \bar{A}$; if the map $\varphi(\alpha)$ mapping $A$ onto $\bar{A}$ is one-to-one, then we call it an isomorphism, denoted by $(A, \circ) \cong(\bar{A}, \bar{\circ})$ or $A \cong \bar{A}$.

\section{Limit Theory of Interval-Valued Intuitionistic Fuzzy Sequences}

To begin with, we point out a fact that in Definition 2 there are four variables in $\widetilde{\mathcal{E}}$, in other words, $\widetilde{\mathcal{E}}=$ $\left(\left(\left[\varepsilon_{1}, \varepsilon_{2}\right],\left[1-\varepsilon_{3}, 1-\varepsilon_{4}\right]\right)\right.$, which is a little tedious. In order to apply the limits in the IVIFC, it is necessary to simplify Definition 2 and give its equivalent counterpart. We present the following result.

Theorem 4. Let $\left\{\widetilde{\alpha}_{n}\right\}$ be an addition sequence of $\widetilde{\alpha}_{0}$, $\lim _{n \longrightarrow+\infty} \widetilde{\alpha}_{n}^{\oplus}=\widetilde{\alpha}_{0}$ if and only if for a given $\operatorname{IFN}(\varepsilon, 1-\varepsilon)$, corresponding to this $\varepsilon$, there exists a positive integer $N$, such that $\tilde{\alpha}_{n} \ominus \tilde{\alpha}_{0}<_{L}(\varepsilon, 1-\varepsilon)$ for $n>N$.

Proof. According to Definition 1, if $\lim _{n \longrightarrow+\infty} \widetilde{\alpha}_{n}^{\oplus}=\widetilde{\alpha}_{0}$, then for a given $\widetilde{\varepsilon}=\left(\left[\varepsilon_{1}, \varepsilon_{2}\right],\left[1-\varepsilon_{3}, 1-\varepsilon_{4}\right]\right)>_{L}(0,1)$, there exists a positive integer $N$, such that $\widetilde{\alpha}_{n} \ominus \widetilde{\alpha}_{0}<_{L}\left(\left[\varepsilon_{1}, \varepsilon_{2}\right]\right.$, [1$\left.\left.\varepsilon_{3}, 1-\varepsilon_{4}\right]\right)$ for $n>N$. If we set $\varepsilon=\max \left\{\varepsilon_{1}, \varepsilon_{2}, \varepsilon_{3}, \varepsilon_{4}\right\}$, then $\left(\left[\varepsilon_{1}, \varepsilon_{2}\right],\left[1-\varepsilon_{3}, 1-\varepsilon_{4}\right]\right)<_{L}(\varepsilon, 1-\varepsilon)$, and thus, $\widetilde{\alpha}_{n} \ominus \widetilde{\alpha}_{0}<_{L}$ $(\varepsilon, 1-\varepsilon)$ for $n>N$.

Conversely, if we put $\varepsilon=\min \left\{\varepsilon_{1}, \varepsilon_{2}, \varepsilon_{3}, \varepsilon_{4}\right\}$, then we have $(\varepsilon, 1-\varepsilon)<_{L}\left(\left[\varepsilon_{1}, \varepsilon_{2}\right],\left[1-\varepsilon_{3}, 1-\varepsilon_{4}\right]\right)$, by assumption, $\widetilde{\alpha}_{n} \ominus \widetilde{\alpha}_{0}<_{L}(\varepsilon, 1-\varepsilon)$ for $n>N$, which implies $\widetilde{\alpha}_{n} \ominus \widetilde{\alpha}_{0}<_{L}$ $\left(\left[\varepsilon_{1}, \varepsilon_{2}\right],\left[1-\varepsilon_{3}, 1-\varepsilon_{4}\right]\right)$, for $n>N$; in view of Definition 2, we obtain that $\lim _{n \longrightarrow+\infty} \widetilde{\alpha}_{n}^{\oplus}=\widetilde{\alpha}_{0}$, which completes the proof.

As described in Introduction, the IFC has its own system and strictly obeys intuitionistic fuzzy operational laws, which is also the most significant characteristic, so is the IVIFC. And thus, we propose the following partial orders rather than the partial order $<_{L}$ in Definition 1 and Theorem 4.

Definition 6. If there is an IVIFN $\tilde{\eta}$, which satisfies $\widetilde{\alpha} \oplus \widetilde{\eta}=\widetilde{\beta}$, then we say that the IVIFN $\widetilde{\alpha}$ is less than or equal to the IVIFN $\widetilde{\beta}$, denoted by $\widetilde{\alpha} \unlhd \widetilde{\beta}$ or $\widetilde{\alpha} \unlhd_{\oplus} \widetilde{\beta}$, and if $\widetilde{\eta} \neq(0,1)$, then we write $\widetilde{\beta} \triangleleft_{\otimes} \widetilde{\alpha}$. If there is an IVIFN $\kappa$, which satisfies $\tilde{\alpha} \otimes \widetilde{\kappa}=\widetilde{\beta}$, then we say that $\widetilde{\beta}$ is less than or equal to $\widetilde{\alpha}$, denoted by $\beta \unlhd_{\oplus} \alpha$, particularly, if $\widetilde{\kappa} \neq(1,0)$, then $\beta \triangleleft_{\otimes} \alpha$.

Definition 6 shows that if $\widetilde{\alpha} \in A_{\widetilde{\alpha}_{0}}^{\oplus}$, then $\widetilde{\alpha}_{0} \unlhd \widetilde{\alpha}$, and if $\tilde{\alpha} \in A_{\tilde{\alpha}_{0}}^{\ominus}$, then $\widetilde{\alpha} \unlhd \widetilde{\alpha}_{0}$. For the multiplication and division regions, $\widetilde{\alpha} \triangleleft_{\otimes} \widetilde{\alpha}_{0}$ holds for $\widetilde{\alpha} \in A_{\widetilde{\alpha}_{0}}^{\otimes}$, and $\widetilde{\alpha}_{0} \unlhd_{\otimes} \widetilde{\alpha}$ is valid for $\widetilde{\alpha} \in A_{\widetilde{\alpha}}^{\otimes}$. If we compare $\unlhd$ with ${ }_{\widetilde{\beta}}<_{L}$, we can see that $\widetilde{\alpha} \unlhd \widetilde{\beta} \Longrightarrow \widetilde{\alpha}<_{L} \widetilde{\beta}$, but $\widetilde{\alpha}<_{L} \widetilde{\beta} \Longrightarrow \widetilde{\alpha} \unlhd \widetilde{\beta}$ does not always hold. In other words, $\widetilde{\alpha} \unlhd \widetilde{\beta}$ means that $\widetilde{\alpha} \in A \stackrel{\ominus}{\ominus}$ or $\widetilde{\beta} \in A_{\alpha}^{\oplus}$. However, when $\widetilde{\alpha}<_{L} \tilde{\beta}, \widetilde{\alpha}$ may not be in $A_{\tilde{\beta}}^{\ominus}$.

Finally, we introduce two symbols $|\bullet|$ and $\langle\bullet\rangle,|\bullet|$ means that if $\widetilde{\alpha}_{n} \in A_{\widetilde{\alpha}_{0}}^{\ominus}$, then $\left|\widetilde{\alpha}_{n} \ominus \widetilde{\alpha}_{0}\right|=\widetilde{\alpha}_{n} \ominus \widetilde{\alpha}_{0}$, and $\left|\widetilde{\alpha}_{n} \ominus \widetilde{\alpha}_{0}\right|=\alpha_{0} \ominus \alpha_{n}$ holds for $\widetilde{\alpha}_{n} \in A_{\widetilde{\alpha}_{0}}^{\oplus} .\langle\bullet\rangle$ implies that if $\tilde{\alpha}_{n} \in A_{\widetilde{\alpha}_{0}}^{\otimes}$, then $\left\langle\widetilde{\alpha}_{n} \oslash \widetilde{\alpha}_{0}\right\rangle=\widetilde{\alpha}_{0} \oslash \widetilde{\alpha}_{n}$, and $\left\langle\widetilde{\alpha}_{n} \oslash \widetilde{\alpha}_{0}\right\rangle=\widetilde{\alpha}_{n} \oslash \widetilde{\alpha}_{0}$ for $\tilde{\alpha}_{n} \in A_{\tilde{\alpha}_{0}}^{\ominus}$. By this technique, we can reduce four kinds of sequences to two kinds: if $\widetilde{\alpha}_{n} \in A_{\widetilde{\alpha}_{0}}^{\oplus} \cup A_{\widetilde{\alpha}_{0}}^{\ominus}$, then we call $\left\{\widetilde{\alpha}_{n}\right\}$ as the first kind of sequence or refer to it as a sequence; if $\widetilde{\alpha}_{n} \in A_{\widetilde{\alpha}_{0}}^{\otimes} \cup A_{\widetilde{\alpha}_{0}}^{\otimes}$, then we say that $\left\{\widetilde{\alpha}_{n}\right\}$ is the second kind of sequence and denote it as $\left\{\widetilde{\alpha}_{n}^{*}\right\}$ so as to distinguish the first kind of sequence.

Based on the above analysis, we can provide a desirable definition with respect to the sequence of the first kind as follows.

Definition 7. Let $\left\{\widetilde{\alpha}_{n}\right\}$ be a sequence of $\widetilde{\alpha}_{0}$, that is, $\widetilde{\alpha}_{n} \in A_{\widetilde{\alpha}_{0}}^{\oplus} \cup A_{\widetilde{\alpha}_{0}}^{\ominus}$. If for a given $\widetilde{\varepsilon}=(\varepsilon, 1-\varepsilon) \triangleright(0,1)$, there exists a positive integer $N$, such that $\left|\widetilde{\alpha}_{n} \ominus \widetilde{\alpha}_{0}\right| \triangleleft \widetilde{\varepsilon}$ for $n>N$, then we say that $\widetilde{\alpha}_{n}$ converges to $\widetilde{\alpha}_{0}$ (or has the limit $\widetilde{\alpha}_{0}$ ), and we write $\lim _{n \longrightarrow+\infty} \widetilde{\alpha}_{n}=\widetilde{\alpha}_{0}$ (or in the concise form $\widetilde{\alpha}_{n} \longrightarrow \widetilde{\alpha}_{0}$ as $n \longrightarrow \infty)$.

Incidentally, similar to the classical calculus, it is obvious that for a given intuitionistic fuzzy sequence, when we omit, 
add, or vary finite many elements of this sequence, the limit of this new sequence is the same if the original sequence converges. Hence, for the convenience of discussion, we omit the words "for all but finitely many $n$ " in Definition 7. Compared with Definition 2, Definition 7 shows that it can better reflect "intuitionistic fuzzy" since the symbol $\triangleleft$ is based on intuitionistic fuzzy operational laws. Moreover, Definition 7 has unified the addition and subtraction limits, and the form is more similar to that of the limit in the classical calculus. Analogously, we can obtain the limit of the second kind. For the sake of simplification, here we omit it.

After a series of preliminary work, in the following, we only need to discuss the limit theory from two aspects rather than four aspects: the sequence of the first kind and the sequence of the second kind.

\subsection{Limit Theory with respect to the Sequence of the First Kind.} As we know, due to the complexity of the intuitionistic fuzzy operational laws, if we directly investigate the limit theory via the intuitionistic fuzzy operational laws, it will be very complex and the computation is very tedious. Particularly, in the interval-valued intuitionistic fuzzy circumstance, it will be more complex and tedious. In the classical calculus, we discuss the limit theory in a metric space: for the one hand, by this technique, we can simplify the computation; for the other hand, we can unify the limit forms of the one-dimensional and the multi-dimensional sequences. So, it is necessary to define the interval-valued intuitionistic fuzzy metric space.

Let $\widetilde{I}$ be a collection of all IVIFNs, for any $\widetilde{\alpha}_{1}=\left(\left[\mu_{11}\right.\right.$, $\left.\left.\mu_{21}\right],\left[v_{11}, v_{21}\right]\right)$ and $\widetilde{\alpha}_{2}=\left(\left[\mu_{12}, \mu_{22}\right],\left[v_{12}, v_{22}\right]\right)$, we define $d\left(\widetilde{\alpha}_{1}, \widetilde{\alpha}_{2}\right)=(1 / 2) \sqrt{\left(\mu_{11}-\mu_{12}\right)^{2}+\left(\mu_{21}-\mu_{22}\right)^{2}+\left(\nu_{11}-v_{12}\right)^{2}+\left(v_{21}-v_{22}\right)^{2}}$. It is clear that $d\left(\widetilde{\alpha}_{1}, \widetilde{\alpha}_{2}\right)$ is a distance measure. Hence, $(\widetilde{I}, d)$ is a metric space, and we call it the interval-valued intuitionistic fuzzy metric space. Based on $(\widetilde{I}, d)$, below we offer an equivalent counterpart of the limit of the first kind:

Theorem 5. Let $\left\{\widetilde{\alpha}_{n}\right\}$ be a sequence of $\widetilde{\alpha}_{0}$ in $(\widetilde{I}, d)$, where $\widetilde{\alpha}_{n}=\left(\left[\mu_{1 n}, \mu_{2 n}\right],\left[v_{1 n}, v_{2 n}\right]\right)$ and $\widetilde{\alpha}_{0}=\left(\left[\mu_{10}, \mu_{20}\right],\left[v_{10}, v_{20}\right]\right)$. $\lim _{n \longrightarrow+\infty} \widetilde{\alpha}_{n}=\widetilde{\alpha}_{0}$ if and only if for every $\varepsilon>0$, there is a positive integer $N$ such that $d\left(\widetilde{\alpha}_{n}, \widetilde{\alpha}_{0}\right)<\varepsilon$ for $n>N$.

Proof. In view of Definition 7, if $\lim _{n \longrightarrow+\infty} \widetilde{\alpha}_{n}=\widetilde{\alpha}_{0}$, then for a given $\varepsilon>0$, there is a positive integer $N$ such that when $n>N$, we have $\left|\mu_{1 n}-\mu_{10}\right|<\left(1-\mu_{10}\right) \varepsilon_{1},\left|\mu_{2 n}-\mu_{20}\right|<$ $\left(1-\mu_{20}\right) \varepsilon_{1},\left|\nu_{1 n}-\nu_{10}\right|<\nu_{10} \varepsilon_{1}$, and $\left|\nu_{2 n}-\nu_{20}\right|<\nu_{20} \varepsilon_{1}$. If we let $\varepsilon=\max \left\{\left(1-\mu_{10}\right) \varepsilon_{1},\left(1-\mu_{20}\right) \varepsilon_{1}, v_{10} \varepsilon_{1}, v_{20} \varepsilon_{1}\right\}$, then $\mid \mu_{1 n}-$ $\mu_{10}|,| \mu_{2 n}-\mu_{20}|,| \nu_{1 n}-v_{10} \mid$, and $\left|\nu_{2 n}-v_{20}\right|$ are all less than $\varepsilon$, which implies that $d\left(\widetilde{\alpha}_{n}, \widetilde{\alpha}_{0}\right)<\varepsilon$ for $n>N$.

Conversely, if $d\left(\widetilde{\alpha}_{n}, \widetilde{\alpha}_{0}\right)<\varepsilon$ for $n>N$, then $\mid \mu_{1 n}-$ $\mu_{10} \mid<2 d\left(\widetilde{\alpha}_{n}, \widetilde{\alpha}_{0}\right)<2 \varepsilon$ for $n>N$, that is, $\left|\mu_{1 n}-\mu_{10} / 1-\mu_{10}\right|$ $<2 \varepsilon / 1-\mu_{10}$. Analogously, when $n>N$, we have $\left|\mu_{2 n}-\mu_{20}\right|$ $1-\mu_{20}\left|<2 \varepsilon / 1-\mu_{20},\right| \nu_{1 n} / \nu_{10} \mid>1-\left(2 \varepsilon / \nu_{10}\right)$, and $\left|\nu_{2 n} / \nu_{20}\right|>1$ $-\left(2 \varepsilon / v_{20}\right)$. Consequently, if we let $\varepsilon^{\prime}=\max \left\{\left(2 \varepsilon / 1-\mu_{10}\right)\right.$, $\left.\left(2 \varepsilon / 1-\mu_{20}\right),\left(2 \varepsilon / \nu_{10}\right),\left(2 \varepsilon / v_{20}\right)\right\}$, then we have $\left|\widetilde{\alpha}_{n} \ominus \widetilde{\alpha}_{0}\right| \triangleleft$ $\left(\varepsilon^{\prime}, 1-\varepsilon^{\prime}\right)$, that is, $\lim _{n \longrightarrow+\infty} \widetilde{\alpha}_{n}=\widetilde{\alpha}_{0}$, which completes the proof.
Theorem 5 shows that when we investigate the limit theory in the interval-valued intuitionistic fuzzy circumstance, we can discuss them in $(\widetilde{I}, d)$. For example, by Theorem 5, we have the following uniqueness property:

Theorem 6. Let $\left\{\widetilde{\alpha}_{n}\right\}$ be a sequence of $\widetilde{\alpha}_{0}$. If $\left\{\widetilde{\alpha}_{n}\right\}$ converges to $\widetilde{\alpha}_{0}$ and $\widetilde{\beta}_{0}$, then $\widetilde{\alpha}_{0}=\widetilde{\beta}_{0}$.

Proof. By Theorem 5, for a given $\varepsilon>0$, there are two positive integers $N_{1}$ and $N_{2}$ such that when $n>N_{1}$, we have $d\left(\widetilde{\alpha}_{n}, \widetilde{\alpha}_{0}\right)<\varepsilon$ and $d\left(\widetilde{\alpha}_{n}, \widetilde{\beta}_{0}\right)<\varepsilon$. If we put $N_{\widetilde{\beta}_{0}}=\lim \left(N_{1}, N_{2}\right)$, then for all $n>N$, we have $d\left(\widetilde{\alpha}_{0}, \widetilde{\beta}_{0}\right) \leq d\left(\widetilde{\alpha}_{n}, \widetilde{\alpha}_{0}\right)+$ $d\left(\widetilde{\alpha}_{n}, \widetilde{\beta}_{0}\right) \leq 2 \varepsilon$. Considering that $\varepsilon$ is arbitrary, we can deduce that $\widetilde{\alpha}_{0}=\widetilde{\beta}_{0}$, which completes the proof.

Theorem 7. Let $\left\{\widetilde{\alpha}_{n}\right\}$ be a sequence of $\widetilde{\alpha}_{0}$, where $\widetilde{\alpha}_{n}=\left(\left[\mu_{1 n}, \mu_{2 n}\right],\left[v_{1 n}, v_{2 n}\right]\right)$ and $\widetilde{\alpha}_{0}=\left(\left[\mu_{10}, \mu_{20}\right],\left[v_{10}, v_{20}\right]\right)$, then $\lim _{n \longrightarrow+\infty} \widetilde{\alpha}_{n}=\widetilde{\alpha}_{0}$ if and only if $\lim _{n \longrightarrow+\infty} \mu_{1 n}=$ $\mu_{10}, \lim _{n \longrightarrow+\infty} \mu_{2 n}=\mu_{20}, \lim _{n \longrightarrow+\infty} \nu_{1 n}=v_{10}$, and $\lim _{n \longrightarrow+\infty}$ $v_{2 n}=v_{20}$.

Proof. According to Theorem 5, if $\lim _{n \longrightarrow+\infty} \widetilde{\alpha}_{n}=\widetilde{\alpha}_{0}$, then for a given $0<\varepsilon \leq 1$, there is a positive integer $N$ such that $d\left(\widetilde{\alpha}_{n}, \widetilde{\alpha}_{0}\right)<\varepsilon$ for $n>N$. Considering that all of $\left|\mu_{1 n}-\mu_{10}\right|,\left|\mu_{2 n}-\mu_{20}\right|,\left|\nu_{1 n}-\nu_{10}\right|$, and $\left|\nu_{2 n}-\nu_{20}\right|$ are less than $2 d\left(\widetilde{\alpha}_{n}, \widetilde{\alpha}_{0}\right)$ for $n>N$, that is, all of them are less than $2 \varepsilon$ for $n>N$, we have $\lim _{n \longrightarrow+\infty} \mu_{1 n}=\mu_{10}, \lim _{n \longrightarrow+\infty} \mu_{2 n}=\mu_{20}$, $\lim _{n \longrightarrow+\infty} v_{1 n}=v_{10}$, and $\lim _{n \longrightarrow+\infty} v_{2 n}=v_{20}$.

Conversely, if the four limits exist, then for a given $\varepsilon>0$, there is a positive integer $N$ such that all of $\left|\mu_{1 n}-\mu_{10}\right|,\left|\mu_{2 n}-\mu_{20}\right|,\left|\nu_{1 n}-v_{10}\right|$, and $\left|\nu_{2 n}-v_{20}\right|$ are less than $\varepsilon$ for $n>N$. Therefore, we have $d\left(\widetilde{\alpha}_{n}, \widetilde{\alpha}_{0}\right)<\varepsilon$ for $n>N$. Using Theorem 5 again, we have $\lim _{n \longrightarrow+\infty} \widetilde{\alpha}_{n}=\widetilde{\alpha}_{0}$, which completes the proof.

It is well-known that when a metric space is not complete, the limit operation may be meaningless. Hence, the completeness is a significant issue in the classical mathematic analysis. For the same reason, suppose that $\left\{\widetilde{\alpha}_{n}\right\}$ is a sequence, if its limit may be not an IVIFN, then a series of problems must arise: the limits of interval-valued intuitionistic fuzzy functions (IVIFFs) at a point maybe not an IVIFN, which leads to that we cannot conveniently discuss the continuity, the derivatives, the differentials, and the indefinite integrals in the IVIFC. Moreover, we cannot conveniently build the definite integrals either, since all of them may be meaningless. Fortunately, we have the following theorem:

Theorem 8. Let $\left\{\widetilde{\alpha}_{n}\right\}$ be a sequence of $\widetilde{\alpha}_{0}$, where $\widetilde{\alpha}_{n}=$ $\left(\left[\mu_{1 n}, \mu_{2 n}\right],\left[v_{1 n}, v_{2 n}\right]\right)$ and $\widetilde{\alpha}_{0}=\left(\left[\mu_{10}, \mu_{20}\right],\left[v_{10}, v_{20}\right]\right)$. If $\lim _{n \longrightarrow+\infty} \widetilde{\alpha}_{n}=\widetilde{\alpha}_{0}$, then the limit $\widetilde{\alpha}_{0}$ is an IVIFN.

Proof. In view of Theorem 7, if $\lim _{n \longrightarrow+\infty} \widetilde{\alpha}_{n}=\widetilde{\alpha}_{0}$, then $\lim _{n \longrightarrow+\infty} \mu_{2 n}=\mu_{20}$ and $\lim _{n \longrightarrow+\infty} v_{2 n}=v_{20}$, since $\left\{\widetilde{\alpha}_{n}\right\}$ is a sequence of IVIFNs, then for each $n$, we have $0 \leq \mu_{2 n}+$ $v_{2 n} \leq 1$ and $\mu_{1 n}, \mu_{2 n}, v_{1 n}, v_{2 n} \in[0,1]$, and thus, $0 \leq \lim _{n \longrightarrow \infty}$ $\left(\mu_{2 n}+v_{2 n}\right) \leq 1,0 \leq \lim _{n \longrightarrow \infty} \mu_{i n} \leq 1$ and $0 \leq \lim _{n \longrightarrow \infty} v_{\text {in }} \leq 1$ for $i=1$, 2. In other words, $0 \leq \mu_{20}+v_{20} \leq 1, \mu_{i 0} \in[0,1]$, and $v_{i 0} \in[0,1]$ for $i=1,2$, that is to say, $\widetilde{\alpha}_{0}$ is an IVIFN, which completes the proof. 
Furthermore, if $\widetilde{\alpha}$ and $\widetilde{\beta}$ are two IVIFNs, Definition 7 implies that $A \stackrel{\alpha_{0}}{\triangleq}=\left\{\widetilde{\alpha}_{0} \diamond \widetilde{\beta} \mid \beta \in \widetilde{I}, \diamond \in\{\oplus, \otimes, \ominus, \oslash\}\right\}$ are all IVIFNs, so are $\hat{\lambda} \tilde{\alpha}$ and $\widetilde{\alpha}^{\lambda}$, we summarize them as a theorem:

Theorem 9. Let $\left\{\widetilde{\alpha}_{n}\right\}$ and $\left\{\widetilde{\beta}_{n}\right\}$ be two sequences, then all of the limits $\tilde{\alpha}_{n} \oplus \widetilde{\beta}_{n}, \widetilde{\alpha}_{n} \ominus \widetilde{\beta}_{n}, \widetilde{\alpha}_{n} \otimes \widetilde{\beta}_{n}, \widetilde{\alpha}_{n} \oslash \widetilde{\beta}_{n}, \lambda \widetilde{\alpha}_{n}$, and $\tilde{\alpha}_{n}^{\lambda}$ are IVIFNs.

Theorem 7 shows that the limit of an interval-valued intuitionistic fuzzy sequence can be transformed into the limits of the membership degrees and the nonmembership degrees. Theorems 8 and 9 indicate that the limit process is closed in interval-valued intuitionistic fuzzy circumstance. Based on which, we have the following desirable properties:

Theorem 10. Let $\left\{\widetilde{\alpha}_{n}\right\}$ and $\left\{\widetilde{\beta}_{n}\right\}$ be two sequences, $\lim _{n \longrightarrow+\infty} \widetilde{\alpha}_{n}=\widetilde{\alpha}_{0}$ and $\lim _{n \longrightarrow+\infty} \widetilde{\beta}_{n}=\widetilde{\beta}_{0}$, then,
(a) $\lim _{n \longrightarrow+\infty}\left(\widetilde{\alpha}_{n} \oplus \widetilde{\beta}_{n}\right)=\widetilde{\alpha}_{0} \oplus \widetilde{\beta}_{0}$
(b) $\lim _{n \longrightarrow+\infty}\left(\widetilde{\alpha}_{n} \ominus \widetilde{\beta}_{n}\right)=\widetilde{\alpha}_{0} \ominus \widetilde{\beta}_{0}$ for $\widetilde{\alpha}_{0} \triangleright \widetilde{\beta}_{0}$
(c) $\lim _{n \longrightarrow+\infty}\left(\widetilde{\alpha}_{n}\right)^{\lambda}=\left(\widetilde{\alpha}_{0}\right)^{\lambda}$, where $\lambda>0$
(d) $\lim _{n \longrightarrow+\infty} \lambda \widetilde{\alpha}_{n}=\lambda \widetilde{\alpha}_{0}$, where $\lambda>0$
(e) $\lim _{n \longrightarrow+\infty}\left(\widetilde{\alpha}_{n} \otimes \widetilde{\beta}_{n}\right)=\widetilde{\alpha}_{0} \otimes \widetilde{\beta}_{0}$
(f) $\lim _{n \longrightarrow+\infty}\left(\widetilde{\alpha}_{n} \oslash \widetilde{\beta}_{n}\right)=\widetilde{\alpha}_{0} \oslash \widetilde{\beta}_{0}$ for $\widetilde{\alpha}_{0} \triangleleft_{\otimes} \tilde{\beta}_{0}$

Proof. We only prove (a), the rest can be proven in the same way.

(a) Let $\widetilde{\alpha}_{n}$ and $\widetilde{\beta}_{n}$ be $\left(\left[\mu_{1 n}, \mu_{2 n}\right],\left[\nu_{1 n}, v_{2 n}\right]\right)$ and $\left(\left[\mu_{1 n}^{\prime}\right.\right.$, $\left.\left.\mu_{2 n}^{\prime}\right],\left[v_{1 n}^{\prime}, v_{2 n}^{\prime}\right]\right)$, respectively. Since $\lim _{n \longrightarrow+\infty} \widetilde{\alpha}_{n}=\widetilde{\alpha}_{0}$ and $\lim _{n \longrightarrow+\infty} \tilde{\beta}_{n}=\widetilde{\beta}_{0}$, then we have $\lim _{n \longrightarrow+\infty}\left(\alpha_{n} \oplus b_{n}\right)=$ $\lim _{n \longrightarrow+\infty}\left(\left[\mu_{1 n}+\mu_{1 n}^{\prime}-\mu_{1 n} \mu_{1 n}^{\prime}, \mu_{2 n}+\mu_{2 n}^{\prime}-\mu_{2 n} \mu_{2 n}^{\prime}\right],\left[\nu_{1 n} \nu_{1 n}^{\prime}\right.\right.$, $\left.\left.v_{2 n} v_{2 n}^{\prime}\right]\right)=\left(\lim _{n \longrightarrow+\infty}\left[\left(\mu_{1 n}+\mu_{1 n}^{\prime}-\mu_{1 n} \mu_{1 n}^{\prime}\right), \lim _{n \longrightarrow+\infty}\left(\mu_{2 n_{\beta^{\prime}}}+\right.\right.\right.$ $\left.\left.\left.\mu_{2 n}^{\prime}-\mu_{2 n} \mu_{2 n}^{\prime}\right)\right],\left[\lim _{n \longrightarrow+\infty} \nu_{1 n} \nu_{1 n}^{\prime}, \lim _{n \longrightarrow+\infty} \nu_{2 n} \nu_{2 n}^{\prime}\right]\right)=\widetilde{\alpha}_{0} \oplus \widetilde{\beta}_{0}$, which completes the proof.

Till now, we have investigated the limit theory with respect to the sequence of the first kind. As described in preliminaries, for an $\operatorname{IVIFN}\left(\mu_{\widetilde{A}}(x), \nu_{\widetilde{A}}(x)\right)$, when $\sup \mu_{\widetilde{A}}(x)=\inf \mu_{\widetilde{A}}(x)$ and $\sup v_{\widetilde{A}}(x)=\inf \nu_{\widetilde{A}}(x)$ for any $x \in X$, the IVIFN are reduced to an IFN, which means that the above results in this subsection also hold for a sequence of IFNs. Indeed, we obtain some theorems, even though we cannot find the corresponding theorems in the IFC either. Hence, these results not only enrich the theory of the IVIFC but also develop the theory of the IFC. For the other hand, the proof methods of these theorems in this subsection are considerably different from those in the existing literature $[16,17,23]$. For example, as for Theorem 7, Zhao et al. [19] did not unify the addition sequence and the subtraction sequence, comparing the corresponding proof in reference [23] with the one of Theorem 7, the latter is considerably simple.

3.2. Limit Theory with respect to the Sequence of the Second Kind. In this subsection, for the sake of simplicity, we only list a conception and two theorems.

Definition 8. Let $\left\{\widetilde{\alpha}_{n}^{*}\right\}$ be a sequence of $\widetilde{\alpha}_{0}$, that is, $\widetilde{\alpha}_{n}^{*} \in A_{\widetilde{\alpha}_{0}}^{\otimes} \cup A_{\widetilde{\alpha}_{0}}^{\otimes}$. If for a given $\widetilde{\varepsilon}=(1-\varepsilon, \varepsilon) \triangleright_{\otimes}(0,1)$, there exists a positive integer $N$, such that $\left\langle\widetilde{\alpha}_{n} \oslash \widetilde{\alpha}_{0}\right\rangle \triangleleft_{\otimes} \widetilde{\varepsilon}$ for $n>N$. In this case, we say that $\widetilde{\alpha}_{n}^{*}$ converges to $\widetilde{\alpha}_{0}$ (or has the limit $\widetilde{\alpha}_{0}$ ), and we write $\lim _{n \longrightarrow+\infty} \widetilde{\alpha}_{n}^{*}=\widetilde{\alpha}_{0}$ (or in the concise form $\widetilde{\alpha}_{n}^{*} \longrightarrow \widetilde{\alpha}_{0}$ as $n \longrightarrow \infty$ ).

Theorem 11. Let $\left\{\widetilde{\alpha}_{n}^{*}\right\}$ and $\left\{\widetilde{\beta}_{n}^{*}\right\}$ be two second kinds of sequences, the following properties are valid:

(a) Let $\left\{\widetilde{\alpha}_{n}^{*}\right\}$ be in $(\widetilde{I}, d), \lim _{n \longrightarrow+\infty} \widetilde{\alpha}_{n}^{*}=\widetilde{\alpha}_{0}$ if and only if for every $\varepsilon>0$, there is a positive integer $N$ such that $d\left(\widetilde{\alpha}_{n}^{*}, \widetilde{\alpha}_{0}\right)<\varepsilon$ for $n>N$

(b) If $\left\{\widetilde{\alpha}_{n}^{*}\right\}$ converges to $\widetilde{\alpha}_{0}$ and $\widetilde{\beta}_{0}$, then the limits $\widetilde{\alpha}_{0}$ and $\tilde{\beta}_{0}$ are IVIFNs and $\widetilde{\alpha}_{0}=\widetilde{\beta}_{0}$

(c) If $\tilde{\alpha}_{n}^{*}=\left(\left[\mu_{1 n}^{*}, \mu_{2 n}^{*}\right],\left[\nu_{1 n}^{*}, v_{2 n}^{*}\right]\right)$ and $\tilde{\alpha}_{0}=\left(\left[\mu_{10}^{*}\right.\right.$, $\left.\left.\mu_{20}^{*}\right],\left[v_{10}^{*}, v_{20}^{*}\right]\right)$, then $\lim _{n \longrightarrow+\infty} \widetilde{\alpha}_{n}^{*}=\widetilde{\alpha}_{0}$ if and only if $\lim _{n \longrightarrow+\infty} \mu_{1 n}^{*}=\mu_{10}, \quad \lim _{n \longrightarrow+\infty} \mu_{2 n}^{*}=\mu_{20}, \quad \lim _{n \longrightarrow+\infty}$ $v_{1 n}^{*}=v_{10}$, and $\lim _{n \longrightarrow+\infty} v_{2 n}^{*}=v_{20}$

(d) All of the limits $\widetilde{\alpha}_{n}^{*} \oplus \widetilde{\beta}_{n}^{*}, \widetilde{\alpha}_{n}^{*} \ominus \widetilde{\beta}_{n}^{*}, \widetilde{\alpha}_{n}^{*} \otimes \widetilde{\beta}_{n}^{*}$, and $\widetilde{\alpha}_{n}^{*} \oslash \widetilde{\beta}_{n}^{*}$ are IVIFNs

Theorem 12. Let $\left\{\widetilde{\alpha}_{n}^{*}\right\}$ and $\left\{\widetilde{\beta}_{n}^{*}\right\}$ be two second kinds of sequences, $\lim _{n \longrightarrow+\infty} \widetilde{\alpha}_{n}^{*}=\widetilde{\alpha}_{0}$ and $\lim _{n \longrightarrow+\infty} \widetilde{\beta}_{n}^{*}=\widetilde{\beta}_{0}$, then,

(a) $\lim _{n \longrightarrow+\infty}\left(\widetilde{\alpha}_{n}^{*} \oplus \tilde{\beta}_{n}^{*}\right)=\widetilde{\alpha}_{0} \oplus \widetilde{\beta}_{0}$

(b) $\lim _{n \longrightarrow+\infty}\left(\widetilde{\alpha}_{n}^{*} \ominus \widetilde{\beta}_{n}^{*}\right)=\widetilde{\alpha}_{0} \ominus \widetilde{\beta}_{0}$ for $\widetilde{\alpha}_{0} \triangleright \widetilde{\beta}_{0}$

(c) $\lim _{n \longrightarrow+\infty}\left(\widetilde{\alpha}_{n}^{*}\right)^{\lambda}=\left(\widetilde{\alpha}_{0}\right)^{\lambda}$, where $\lambda>0$

(d) $\lim _{n \longrightarrow+\infty} \lambda \widetilde{\alpha}_{n}^{*}=\lambda \widetilde{\alpha}_{0}$, where $\lambda>0$

(e) $\lim _{n \longrightarrow+\infty}\left(\widetilde{\alpha}_{n}^{*} \otimes \widetilde{\beta}_{n}^{*}\right)=\widetilde{\alpha}_{0} \otimes \widetilde{\beta}_{0}$

(f) $\lim _{n \longrightarrow+\infty}\left(\widetilde{\alpha}_{n}^{*} \oslash \widetilde{\beta}_{n}^{*}\right)=\widetilde{\alpha}_{0} \oslash \widetilde{\beta}_{0}$ for $\widetilde{\alpha}_{0} \triangleleft_{\otimes} \widetilde{\beta}_{0}$

\section{Limit and Continuity Theory of IVIFFs}

To begin with, let us recall Definition 3. The authors defined $F(\tilde{X})=\left(\left[f_{1}\left(\mu_{1}, \mu_{2}, v_{1}, v_{2}\right), f_{2}\left(\mu_{1}, \mu_{2}, v_{1}, v_{2}\right)\right],\left[g_{1}\left(\mu_{1}, \mu_{2}, v_{1}\right.\right.\right.$, $\left.\left.\left.v_{2}\right), g_{2}\left(\mu_{1}, \mu_{2}, v_{1}, v_{2}\right)\right]\right)$, and in Theorem 1 , the authors pointed out that if an IVIFF is differentiable at a point, then the IVIFF has the form $F(\tilde{X})=\left(\left[f_{1}\left(\mu_{1}\right), f_{2}\left(\mu_{2}\right)\right],\left[g_{1}\left(\nu_{1}\right), g_{2}\left(\nu_{2}\right)\right]\right)$, where $\tilde{X}=\left(\left[\mu_{1}, \mu_{2}\right],\left[\nu_{1}, \nu_{2}\right]\right)$. Hence, in our opinion, it is appropriate to define the IVIFF $F(\tilde{X})=\left(\left[f_{1}\left(\mu_{1}\right), f_{2}\left(\mu_{2}\right)\right]\right.$, $\left.\left[g_{1}\left(\nu_{1}\right), g_{2}\left(\nu_{2}\right)\right]\right)$, which can unify the form of the IVIFF. For brevity, we write $(f(\widetilde{\mu}), g(\widetilde{\nu}))$ instead of $\left(\left[f_{1}\left(\mu_{1}\right), f_{2}\left(\mu_{2}\right)\right]\right.$, $\left.\left[g_{1}\left(\nu_{1}\right), g_{2}\left(\nu_{2}\right)\right]\right)$ without causing confusion.

Analogous to Section 3, we can discuss the limit and continuity theories of IVIFFs from two aspects if we use the symbols $|\bullet|$ and $\langle\bullet\rangle$.

4.1. Limit and Continuity Theory of IVIFFs for the First Kind. In modern mathematics, the set is a significant tool. Hence, below we provide the definition for the limit of an IVIFF which is different from the existing literature $[16,17,23]$ :

Definition 9. Let $F(\widetilde{X}): D \longrightarrow \widetilde{I}$ be an IVIFF, and $\widetilde{X}_{0}$ be an accumulation point of $D$. If for any given $0<\varepsilon \leq 1$, there exists an IVIFN $\widetilde{\delta} \triangleright(0,1)$, such that $|F(\widetilde{X}) \ominus \widetilde{A}| \triangleleft(\varepsilon, 1-\varepsilon)$ holds for $\widetilde{X} \in D \cap \stackrel{o}{U}\left(\widetilde{X}_{0}, \widetilde{\delta}\right)$, where $\stackrel{o}{U}\left(\widetilde{X}_{0}, \widetilde{\delta}\right)$, the deleted 
neighborhood of $\widetilde{X}_{0}$, is $(0,1) \triangleleft\left|\widetilde{X} \ominus \widetilde{X}_{0}\right| \triangleleft \widetilde{\delta}$; then we call that $F(\widetilde{X})$ converges to $\widetilde{A}$ (or has the limit $\widetilde{A}$ ) as $\widetilde{X}$ tends to $\widetilde{X}_{0}$, which is denoted by $\lim _{\tilde{X}} \underset{\widetilde{X}}{\longrightarrow} F(\underset{O}{\tilde{X}})=\widetilde{A}$.

It should be noted that $\stackrel{\widetilde{X}}{\rightarrow} \in D \cap U\left(\widetilde{\alpha}_{0}, \widetilde{\delta}\right)$ rather than $\widetilde{X} \in U\left(\widetilde{\alpha}_{0}, \widetilde{\delta}\right)$, for instance, $F(\widetilde{X})$ is defined over $D=\left(\left[\mu_{1}\right.\right.$, $\left.\left.\mu_{2}\right],\left[v_{1}, v_{2}\right]\right)$, where $\mu_{1}, \mu_{2}, v_{1}$, and $v_{2}$ are all rational numbers, then $\widetilde{X} \in U\left(\widetilde{\alpha}_{0}, \delta\right)$ means that $\mu_{1}, \mu_{2}, v_{1}$, and $\nu_{2}$ can be irrational numbers. Obviously, in this case, $|F(\widetilde{X}) \ominus \widetilde{A}| \triangleleft(\varepsilon, 1-\varepsilon)$ is meaningless since $F(\widetilde{X})$ is meaningless. Furthermore, in Definition 9 we have unified the forms of the addition and subtraction limits via the symbol $|\bullet|$. Incidentally, we can follow the ideas in Section 3 to obtain a series of corresponding properties with respect to the limits of the IVIFFs, in other words, Theorems 5-10 also hold for the IVIFFs. For the sake of brevity, here we omit them.

Based on Definition 9, we offer the notions of the interval-valued IFI (IVIFI) and its order and then investigate its basic properties.

Definition 10. Let $F(\widetilde{X})$ be an IVIFF. If $\lim _{\tilde{X}} \longrightarrow \tilde{X}_{0}$ $F(\widetilde{X})=(0,1)$, then $F(\tilde{X})$ is said to be an IVIFI (or infinitely small) at $\tilde{X}_{0}$; let $F_{1}(\tilde{X})$ and $F_{2}(\tilde{X})$ be two IVIFIs at $\tilde{X}_{0}$. If $\lim _{\widetilde{X} \longrightarrow \tilde{X}_{0}}\left(F_{1}(\widetilde{X}) / F_{2}(\tilde{X})\right)=(0,1)$, then we say that $F_{1}(\widetilde{X})$ is IVIFI of a higher order at $\widetilde{X}_{0}$ than $F_{2}(\widetilde{X})$; denoted by $F_{1}(\tilde{X})=o\left(F_{2}(\tilde{X})\right)$; if $\lim _{\tilde{X} \longrightarrow \widetilde{X}_{0}}\left(F_{1}(\tilde{X}) / F_{2}(\tilde{X})\right)=(1,0)$, then $F_{1}(\widetilde{X})$ and $F_{2}(\widetilde{X})$ are equivalent IVIFIs at $\widetilde{X}_{0}$, denoted by $F_{1}(\widetilde{X}) \sim F_{2}(\widetilde{X})$.

Theorem 13. Let $F_{1}(\tilde{X})$ and $F_{2}(\tilde{X})$ be two IVIFIs at $\tilde{X}_{0}$, where $\quad F_{1}(\tilde{X})=\left(\left[f_{11}\left(\mu_{1}\right), f_{21}\left(\mu_{2}\right)\right],\left[g_{11}\left(v_{1}\right), g_{21}\left(v_{2}\right)\right]\right)$ and $F_{2}(\tilde{X})=\left(\left[f_{12}\left(\mu_{1}\right), f_{22}\left(\mu_{2}\right)\right],\left[g_{12}\left(\nu_{1}\right), g_{22}\left(v_{2}\right)\right]\right)$. If $F_{1}(\widetilde{X})=o\left(F_{2}(\widetilde{X})\right)$ at $\widetilde{X}_{0}$, then $F_{1}(\widetilde{X})=\left(\left[o\left(f_{12}\left(\mu_{1}\right)\right)\right.\right.$, $\left.\left.o\left(f_{22}\left(\mu_{2}\right)\right)\right], \quad\left[1-o\left(1-g_{12}\left(v_{1}\right)\right), 1-o\left(1-g_{22}\left(v_{2}\right)\right)\right]\right) ;$ if $F(\widetilde{X}) \sim G(\widetilde{X})$ at $\tilde{X}_{0}$, then $f_{i 1} \sim f_{i 2}, g_{i 1}=g_{i 2}+o\left(1-g_{i 2}\right)$ for $i=1,2$.

Proof. Since $F_{1}(\tilde{X})=o\left(F_{2}(\tilde{X})\right)$ at $\tilde{X}_{0}$, then $\lim _{\tilde{X}} \longrightarrow \tilde{X}$ $\left(F_{1}(\tilde{X}) / F_{2}(\tilde{X})\right)=(0,1)$, that is to say, $\lim _{\mu_{i} \longrightarrow \mu_{i 0}}\left(f_{i 1}^{X}\left(\mu_{i}\right)\right)$ $\left.f_{i 2}\left(\mu_{i}\right)\right)=0$ and $\lim _{v_{i}} \rightarrow v_{i 0}\left(g_{i 1}\left(v_{i}\right)-g_{i 2}\left(v_{i}\right) / 1-g_{i 2}\left(v_{i}\right)\right)=1$ for $i=1,2$. For the former, it is obvious that $f_{i 1}\left(\mu_{i}\right)=o\left(f_{i 2}\right.$ $\left.\left(\mu_{i}\right)\right)$ for $i=1,2$; for the latter, we have $\lim _{v_{i} \longrightarrow v_{i 0}}$ $\left(1-g_{i 1}\left(v_{i}\right) / 1-g_{i 2}\left(v_{i}\right)\right)=0,(i=1,2)$. Thus, we conclude that $1-g_{i 1}\left(v_{i}\right)=o\left(1-g_{i 2}\left(v_{i}\right)\right)$ for $i=1$, 2, which implies that $g_{i 1}\left(v_{i}\right)=1-o\left(1-g_{i 2}\left(v_{i}\right)\right)$ for $i=1$, 2. As for $F(\widetilde{X}) \sim$ $G(\widetilde{X})$ at $\widetilde{X}_{0}$, we can prove it in the same way, here we omit it, which completes the proof.

Theorem 13 reveals the concrete forms concerning the higher order and the equivalently infinitesimals, based on which we can deduce the following results:

Theorem 14. If $F(\tilde{X})$ is an IVIFI at $\widetilde{X}_{0}$, then $o(F(\tilde{X})) \oplus$ $o(F(\widetilde{X}))=o(F(\widetilde{X}))$; if $G(\widetilde{X})=o(F(\widetilde{X}))$, then $o(F(\widetilde{X})) \oplus$ $o(G(\widetilde{X}))=o(F(\widetilde{X}))$.

The following theorem reveals why we call the two IVIFIs to be equivalent IVIFIs.
Theorem 15. Let $F_{1}(\widetilde{X}) \sim F_{2}(\widetilde{X})$ and $G_{1}(\widetilde{X}) \sim G_{2}(\widetilde{X})$ at $\widetilde{X}_{0}$, and $H(\widetilde{X})$ is meaningful in $\stackrel{o}{U}\left(\widetilde{X}_{0}\right)$. If the limits of $F_{1}(\tilde{X}) / F_{2}(\tilde{X})$ and $G_{1}(\tilde{X}) / G_{2}(\tilde{X})$ exist at $\tilde{X}_{0}$, then $\lim _{\tilde{X}} \longrightarrow \tilde{X}_{0}$ $\left(F_{1}(\tilde{X}) / G_{1}(\tilde{X})\right)=\lim _{\tilde{X} \longrightarrow \tilde{X}_{0}}\left(F_{2}(\tilde{X}) / G_{2}(\tilde{X})\right) ; \quad$ if $\lim _{\tilde{X} \longrightarrow \tilde{X}_{0}}$ $\left(F_{1}(\widetilde{X}) \otimes H(\widetilde{X})\right)=\widetilde{A}$, then $\lim _{\widetilde{X} \longrightarrow \widetilde{X}_{0}}\left(F_{2}(\widetilde{X}) \otimes H(\widetilde{X})\right)=\widetilde{A}$.

Proof. $\quad \lim _{\tilde{X} \longrightarrow \tilde{X}_{0}}\left(F_{1}(\tilde{X}) / G_{1}(\tilde{X})\right)=\lim _{\tilde{X}} \longrightarrow \widetilde{X}_{0}\left(\left(F_{1}(\tilde{X}) /\right.\right.$ $\left.\left.G_{1}(\widetilde{X})\right) \otimes\left(F_{2}(\tilde{X}) / F_{1}(\tilde{X})\right) \otimes\left(G_{1}(\widetilde{X}) / G_{2}(\widetilde{X})\right)\right)=\lim _{\tilde{X}} \longrightarrow \tilde{X}_{0}$ $\left(\left(G_{1}(\tilde{X}) / G_{1}(\widetilde{X})\right) \otimes\left(F_{1}(\widetilde{X}) / F_{1}(\widetilde{X})\right) \otimes\left(F_{2}(\tilde{X}) / G_{2}(\tilde{X})\right)\right)=$ $\lim _{\tilde{X} \longrightarrow \tilde{X}_{0}}\left(F_{2}(\tilde{X}) / G_{2}(\tilde{X})\right)$; Since $\lim _{\tilde{X}} \rightarrow \widetilde{X}_{0} F_{1}(\tilde{X}) \otimes H(\tilde{X})=$ $\lim _{\tilde{X}} \longrightarrow \tilde{X}_{0}\left(F_{1}(\tilde{X}) / F_{2}(\tilde{X})\right) \otimes F_{2}(\widetilde{X}) \otimes H(\widetilde{X})=(1,0) \otimes \lim _{\tilde{X}} \longrightarrow \tilde{X}_{0}$ $\left(F_{2}(\vec{X}) \otimes H(\widetilde{X})\right)=\widetilde{A}$, then we have $\lim _{\tilde{X}} \longrightarrow \tilde{X}_{0} F_{2}(\widetilde{X}) \otimes$ $H(\widetilde{X})=\widetilde{A}$, which completes the proof.

Subsequently, we reveal the relationship between the IVIFI and the limit.

Theorem 16. Let $F(\widetilde{X})$ be an IVIFF. $\lim _{\tilde{X}} \longrightarrow \tilde{X}_{0} F(\widetilde{X})=\widetilde{A}$ if and only if $|F(\widetilde{X}) \ominus \widetilde{A}|=\widetilde{\alpha}$, where $\widetilde{\alpha}$ is an IVIFI at $\widetilde{X}_{0}$.

The proof of Theorem 13 is obvious, here we omit it.

As we know, the continuity of a function is based on the notion of the limit. In what follows, we offer the concept of the continuity in the interval-valued intuitionistic fuzzy circumstance.

Definition 11. Let $F(\widetilde{X}): D \longrightarrow \widetilde{I}$ be an IVIFF, and $\widetilde{X}_{0} \in D$ $\left(\widetilde{X}_{0}\right.$ can be an accumulation point of $D$ or be an isolated point of $D)$. If for any given $0<\varepsilon \leq 1$, there exists an IVIFN $\tilde{\delta} \triangleright(0$, $1)$, such that $\left|F(\widetilde{X}) \ominus F\left(\widetilde{X}_{0}\right)\right| \triangleleft(\varepsilon, 1-\varepsilon)$ holds for $\tilde{X} \in D$ $\cap U\left(\widetilde{X}_{0}, \widetilde{\delta}\right)$; then we say that the $\operatorname{IVIFF} F(\tilde{X})$ is continuous at $\widetilde{X}_{0}$ in $D$. We also say that $F(\widetilde{X})$ is continuous at $\widetilde{X}_{0}$ without causing confusion. If $F(\tilde{X})$ is continuous at each point of the set $D$, then we say that $F(\tilde{X})$ is continuous on $D$.

Definition 11 indicates that if $F(\widetilde{X})$ is continuous at an accumulation point $\widetilde{X}_{0}$ in $D$, then $\lim _{\tilde{X}} \longrightarrow \widetilde{X}_{0} F(\widetilde{X})=F\left(\widetilde{X}_{0}\right)$. If $\widetilde{X}_{0}$ is an isolated point of $D$, then it is clear that $\left|F(\widetilde{X}) \ominus F\left(\widetilde{X}_{0}\right)\right| \triangleleft(\varepsilon, 1-\varepsilon)$ holds; in other words, $F(\widetilde{X})$ is continuous at any isolated point.

As mentioned above, Theorems 5-10 also hold for the IVIFFs, and therefore, we have the following theorem:

Theorem 17. If the IVIFFs $F(\widetilde{X})$ and $G(\widetilde{X})$, defined on a set $D$, are both continuous at $\widetilde{X}_{0} \in D$, then all of the IVIFFs $F(\tilde{X}) \oplus G(\tilde{X}), \quad F(\tilde{X}) \ominus G(\tilde{X}) \quad$ for $F(\tilde{X}) \triangleright G(\tilde{X})), \quad F(\tilde{X}) \otimes$ $G(\tilde{X})$, and $F(\tilde{X}) \oslash G(\widetilde{X})\left(\right.$ for $\left.F(\widetilde{X}) \triangleleft_{\otimes} G(\widetilde{X})\right)$ are continuous at $\widetilde{X}_{0}$.

We end this subsection with the intermediate value theorem to show that the IVIFC has its own system.

Theorem 18. If $F(\widetilde{X})$ is continuous on the closed intuitionistic fuzzy interval $\left[\widetilde{\alpha}_{0}, \widetilde{\beta}_{0}\right]=\left\{\widetilde{\gamma} \mid \widetilde{\alpha}_{0} \triangleleft \widetilde{\gamma} \triangleleft \widetilde{\beta}_{0}\right\}$, if $F\left(\widetilde{\alpha}_{0}\right) \neq$ $F\left(\widetilde{\beta}_{0}\right)$ and $\tilde{\chi}$ is an arbitrary IVIFN with $F\left(\widetilde{\alpha}_{0}\right) \triangleleft \widetilde{\chi} \triangleleft F\left(\widetilde{\beta}_{0}\right)$, then there is at least one IVIFN $\widetilde{\xi}\left(\widetilde{\xi} \in\left(\widetilde{\alpha}_{0}, \widetilde{\beta}_{0}\right)\right)$ such that $F(\widetilde{\xi})=\widetilde{\chi}$. 
Proof. For simplicity, we only consider a particular case. The IVIFNs reduce to the IFNs; in the interval-valued intuitionistic fuzzy circumstance, we can prove the result in the same manner. In the intuitionistic fuzzy circumstance, $F\left(\widetilde{\alpha}_{0}\right)$ and $F\left(\widetilde{\beta}_{0}\right)$ become $F\left(\alpha_{0}\right)$ and $F\left(\beta_{0}\right)$, respectively, the others can be expressed in the same manner.

Without loss of generality, assume that $F\left(\alpha_{0}\right) \triangleleft F\left(\beta_{0}\right)$ and $F\left(\alpha_{0}\right) \triangleleft \chi \triangleleft F\left(\beta_{0}\right)$, we define $I_{0}=\left[\alpha_{0}, \beta_{0}\right]$ and $I_{n}=\left[\alpha_{n}, \beta_{n}\right](n=1,2,3, \ldots)$ in the following; on the one hand, we show how to define $I_{n+1}$ if we have defined $I_{n}$; on the other hand, we also show how to seek out the point $\widetilde{\xi}$.

As shown in Figure 2, if $F\left(P_{0}\right)=\chi$, then we only take $\xi=P_{0}$. Otherwise, we decompose $\left[\alpha_{0}, \beta_{0}\right]$ into four parts: $S_{1}, S_{2}, S_{3}$, and $S_{4} . \xi$ should be in one of the regions $S_{1}, S_{2}, S_{3}$, and $S_{4}$ since $F\left(\alpha_{0}\right) \triangleleft \chi \triangleleft F\left(\beta_{0}\right)$ and $F(\xi)=\chi$. Let us assume that $\xi$ is in $S_{2}$; in this case, we can take $\left[\alpha_{1}, \beta_{1}\right]=S_{2}$ with $F\left(\alpha_{1}\right) \triangleleft \chi \triangleleft F\left(\beta_{1}\right)$. Repeating the process, we can get a nested closed interval $I_{n}=\left[\alpha_{n}, \beta_{n}\right](n=0,1,2, \cdots)$. Theorem 2 shows that there exists an IFV $\xi$ such that $\xi \in\left[\alpha_{n}, \beta_{n}\right], \quad(n=$ $0,1,2, \cdots)$ and $\xi=\lim _{n \longrightarrow \infty} \alpha_{n}=\lim _{n \longrightarrow \infty} \beta_{n}$. Finally, we should prove $F(\xi)=\chi$. In view of the continuity of $F(\tilde{X})$, we have $f(\xi)=\lim _{n \longrightarrow \infty} f\left(\alpha_{n}\right)$; it follows from $F\left(\alpha_{n}\right) \triangleleft \chi$ that $\lim _{n \longrightarrow \infty} f\left(\alpha_{n}\right) \unlhd \chi$, that is, $f(\xi) \unlhd \chi$. On the other hand, $f(\xi) \triangleright \chi$ can be obtained if we investigate the sequence $f\left(\beta_{n}\right)$. Consequently, $F(\xi)=\chi$, which completes the proof.

The proof of Theorem 18 shows that it is more complicated than the corresponding proof in the classical mathematics analysis. Particularly, it is interesting and key to decompose the domain of $F(\tilde{X})$. The complexities of intuitionistic fuzzy operational laws lead to those of the IFC; we cannot take it for granted that the corresponding result holds in the IFC if the result is valid in the classical calculus [13] even though sometimes they seem to be very similar. Since they are different in essence, we must offer a rigid proof when we investigate a property which seems to be true.

4.2. Limit and Continuity Theory of IVIFFs with respect to the Second Kind. In this subsection, for the sake of simplicity, two conceptions and a theorem are listed.

Definition 12. Let $F\left(\widetilde{X}^{*}\right): D \longrightarrow \widetilde{I}$ be the second kind of IVIFF, and $\tilde{X}_{0}$ be an accumulation point of $D$. If for any given $0<\varepsilon \leq 1$, there exists an $\operatorname{IVIFN} \widetilde{\delta} \triangleright(0,1)$, such that $\left\langle F\left(\widetilde{X}^{*}\right) \oslash \widetilde{A}\right\rangle \triangleleft_{\otimes}(1-\varepsilon, \varepsilon)$ holds for $\widetilde{X}^{*} \in D \cap \stackrel{o}{\Theta}_{\otimes}\left(\widetilde{X}_{0}, \widetilde{\delta}\right)$, where $\stackrel{o}{U}_{\otimes}\left(\widetilde{X}_{0}, \widetilde{\delta}\right)$, the deleted multiplication and division neighborhood of $\widetilde{X}_{0}$, is $\left\langle\widetilde{X} \oslash \widetilde{X}_{0}\right\rangle \triangleleft_{\otimes} \widetilde{\delta}$; then, we call that $F\left(\widetilde{X}^{*}\right)$ converges to $\widetilde{A}$ with respect to the multiplication and the division (or has the limit $\widetilde{A}$ ) as $\tilde{X}^{*}$ tends to $\widetilde{X}_{0}$, which is denoted by $\lim _{\widetilde{X}^{*} \longrightarrow \widetilde{X}_{0}} F\left(\widetilde{X}^{*}\right)=\widetilde{A}$.

If $\lim _{\widetilde{X}^{*} \longrightarrow \tilde{X}} F\left(\tilde{X}^{*}\right)=F\left(\tilde{X}_{0}\right)$ holds for $\tilde{X}_{0} \in D\left(\tilde{X}_{0}\right.$ can be an accumulation point of $D$ or be an isolated point of $D)$, then we say that the IVIFF $F\left(\tilde{X}^{*}\right)$ is the multiplication and the division continuous at $\widetilde{X}_{0}$ in $D$. We also say that $F\left(\widetilde{X}^{*}\right)$ is continuous at $\tilde{X}_{0}$ without causing confusion. If $F\left(\tilde{X}^{*}\right)$ is continuous at each point of the set $D$, then we say that $F\left(\widetilde{X}^{*}\right)$ is continuous on $D$.

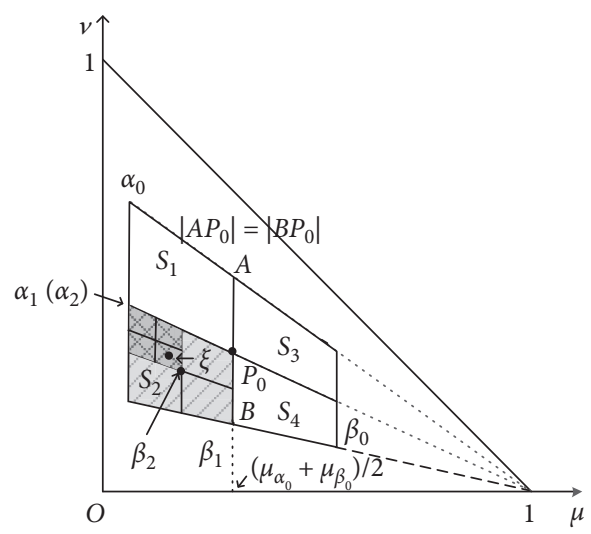

FIgURE 2: Geometric interpretation of Theorem 18.

Definition 13. Let $F\left(\widetilde{X}^{*}\right)$ be an IVIFF of $\widetilde{X}^{*}$. If $\lim _{\tilde{X} \longrightarrow \tilde{X}_{0}^{*}} F\left(\tilde{X}^{*}\right)=(1,0)$, then $F\left(\tilde{X}^{*}\right)$ is said to be an IVIFI with respect to the multiplication and division at $\tilde{X}_{0}^{*}$. Let $F_{1}$ $\left(\tilde{X}^{*}\right)$ and $F_{2}\left(\tilde{X}^{*}\right)$ be two IVIFIs at $\tilde{X}_{0}$. If $\lim _{\tilde{X}^{*} \longrightarrow} \tilde{X}_{0}$ $\left(F_{1}\left(\tilde{X}^{*}\right) \ominus F_{2}\left(\tilde{X}^{*}\right)\right)=(1,0)$, then we say $F_{1}(\tilde{X})$ is IVIFI of a higher order at $\widetilde{X}_{0}$ than $F_{2}(\widetilde{X})$; denoted by $F_{1}\left(\widetilde{X}^{*}\right)=o$ $\left(F_{2}\left(\tilde{X}^{*}\right)\right)$; if $\lim _{\widetilde{X}^{*} \longrightarrow \tilde{X}_{0}}\left(F_{1}\left(\tilde{X}^{*}\right) \ominus F_{2}\left(\tilde{X}^{*}\right)\right)=(0,1)$, then $F_{1}\left(\tilde{X}^{*}\right)$ and $F_{2}\left(\tilde{X}^{*}\right)$ are equivalently intuitionistic fuzzy, infinitely small at $\widetilde{X}_{0}$, denoted by $F_{1}\left(\tilde{X}^{*}\right) \sim F_{2}\left(\widetilde{X}^{*}\right)$.

Theorem 19. The following results are valid:

(a) Let $F_{1}\left(\widetilde{X}^{*}\right)$ and $F_{2}\left(\widetilde{X}^{*}\right)$ be two IVIFIs at $\widetilde{X}_{0}$, where $F_{1}\left(\tilde{X}^{*}\right)=\left(\left[f_{11}\left(\mu_{1}^{*}\right), f_{21}\left(\mu_{2}^{*}\right)\right],\left[g_{11}\left(\nu_{1}^{*}\right), g_{21}\left(v_{2}^{*}\right)\right]\right)$ and $F_{2}\left(\widetilde{X}^{*}\right)=\left(\left[f_{12}\left(\mu_{1}^{*}\right), f_{22}\left(\mu_{2}^{*}\right)\right],\left[g_{12}\left(\nu_{1}^{*}\right), g_{22}\right.\right.$ $\left.\left.\left(v_{2}^{*}\right)\right]\right)$; if $F_{1}\left(\widetilde{X}^{*}\right)=o\left(F_{2}\left(\widetilde{X}^{*}\right)\right)$ at $\widetilde{X}_{0}$, then $F_{1}\left(\widetilde{X}^{*}\right)=$ $\left(\left[1-o\left(1-f_{12}\left(\mu_{1}^{*}\right)\right), 1-o\left(1-f_{22}\left(\mu_{2}^{*}\right)\right)\right],\left[o\left(g_{12}\right.\right.\right.$ $\left.\left.\left.\left(v_{1}^{*}\right)\right), o\left(g_{22}\left(v_{2}^{*}\right)\right)\right]\right)$; if $F\left(\widetilde{X}^{*}\right) \sim G\left(\widetilde{X}^{*}\right)$ at $\widetilde{X}_{0}$, then $f_{i 1}=f_{i 2}+o\left(1-f_{i 2}\right)$, and $g_{i 1} \sim g_{i 2}$ for $i=1,2$.

(b) If $F\left(\widetilde{X}^{*}\right)$ is an IVIFI at $\widetilde{X}_{0}$, then $o\left(F\left(\widetilde{X}^{*}\right)\right) \otimes o$ $\left(F\left(\tilde{X}^{*}\right)\right)=o\left(F\left(\widetilde{X}^{*}\right)\right)$; if $G\left(\tilde{X}^{*}\right)=o\left(F\left(\widetilde{X}^{*}\right)\right)$, then $o\left(F\left(\tilde{X}^{*}\right)\right) \otimes o\left(G\left(\tilde{X}^{*}\right)\right)=o\left(F\left(\tilde{X}^{*}\right)\right)$.

(c) Let $F_{1}\left(\tilde{X}^{*}\right) \sim F_{2}\left(\tilde{X}^{*}\right)$ and $G_{1}\left(\tilde{X}^{*}\right) \sim G_{2}\left(\tilde{X}^{*}\right)$ at $\tilde{X}_{0}$ and $H\left(\widetilde{X}^{*}\right)$ is meaningful in $\stackrel{o}{U}\left(\widetilde{X}_{0}\right)$. If the limits of $F_{1}\left(\tilde{X}^{*}\right) \ominus F_{2}\left(\tilde{X}^{*}\right)$ and $G_{1}\left(\widetilde{X}^{*}\right) \ominus G_{2}\left(\widetilde{X}^{*}\right)$ exist at $\widetilde{X}_{0}$, then $\lim _{\tilde{X}^{*} \longrightarrow \tilde{X}_{0}}\left(F_{1}\left(\widetilde{X}^{*}\right) \ominus F_{2}\left(\tilde{X}^{*}\right)\right)=\lim _{\tilde{X}^{*} \longrightarrow \tilde{X}_{0}}\left(G_{1}\right.$ $\left.\left(\widetilde{X}^{*}\right) \ominus G_{2}\left(\tilde{X}^{*}\right)\right)$. And if $\lim _{\tilde{X}^{*}} \longrightarrow \tilde{X}_{0}\left(F_{1}\left(\widetilde{X}^{*}\right) \oplus H\right.$ $\left.\left(\tilde{X}^{*}\right)\right)=\widetilde{A}$, then $\lim _{\tilde{X}^{*}} \longrightarrow \tilde{X}_{0}\left(F_{2}\left(\tilde{X}^{*}\right) \oplus H\left(\tilde{X}^{*}\right)\right)=\widetilde{A}$.

(d) Let $F\left(\widetilde{X}^{*}\right)$ be an IVIFF. $\lim _{\tilde{X}^{*}} \longrightarrow \tilde{X}_{0} F\left(\widetilde{X}^{*}\right)=\widetilde{A}$ if and only if $\langle F(\tilde{X}) \oslash \widetilde{A}\rangle=\widetilde{\alpha}$, where $\widetilde{\alpha}_{0}$ is an IVIFI with respect to the multiplication and division at $\tilde{X}_{0}$.

(e) If the IVIFFs $F\left(\tilde{X}^{*}\right)$ and $G\left(\tilde{X}^{*}\right)$, defined on a set $D$, are both continuous at $\widetilde{X}_{0} \in D$, then all of the IVIFFs $F\left(\widetilde{X}^{*}\right) \oplus G\left(\widetilde{X}^{*}\right), F\left(\widetilde{X}^{*}\right) \ominus G\left(\widetilde{X}^{*}\right)($ for $F(\widetilde{X}) \unrhd G(\tilde{X}))$, $F\left(\tilde{X}^{*}\right) \otimes G\left(\tilde{X}^{*}\right)$, and $F\left(\tilde{X}^{*}\right) \oslash G\left(\tilde{X}^{*}\right)$ (for $F\left(\widetilde{X}^{*}\right)$ $\left.\unlhd_{\otimes} G\left(\widetilde{X}^{*}\right)\right)$ are continuous at $\widetilde{X}_{0}$.

(f) If $F\left(\tilde{X}^{*}\right)$ is continuous on the closed intuitionistic fuzzy interval $\left[\widetilde{\alpha}_{0}, \widetilde{\beta}_{0}\right]_{\otimes}=\left\{\tilde{\gamma} \mid \widetilde{\alpha}_{0} \triangleleft_{\otimes} \widetilde{\gamma} \triangleleft_{\otimes} \widetilde{\beta}_{0}\right\}$. If $F\left(\widetilde{\alpha}_{0}\right)$ $\neq F\left(\widetilde{\beta}_{0}\right)$ and $\tilde{\chi}$ is an arbitrary IVIFN with $F\left(\widetilde{\alpha}_{0}\right)$ 
$\unlhd_{\otimes} \widetilde{X} \unlhd_{\otimes} F\left(\tilde{\beta}_{0}\right)$, then there is at least one IVIFN $\tilde{\xi}$ $\left.\widetilde{(\xi} \in\left(\widetilde{\alpha}_{0}, \widetilde{\beta}_{0}\right)\right)$ such that $F(\widetilde{\xi})=\tilde{\chi}$.

\section{Differential Theory of IVIFFs}

In the existing literature $[16,23]$, the notions of the derivatives were first offered and then the differentials were introduced. Indeed, in the classical textbook of mathematics analysis, the notion of differential was first given and then the derivative was discussed, which can better reflect the history of the calculus. Motivated by this, in this section, we shall first provide the concepts of the differentials regarding IVIFFs and then investigate their derivatives.

Definition 14. An IVIFF $F(\widetilde{X})=\left(\left[f_{1}\left(\mu_{1}\right), f_{2}\left(\mu_{2}\right)\right]\right.$, $\left.\left[g_{1}\left(\nu_{1}\right), g_{2}\left(v_{2}\right)\right]\right)$ is defined on a set $D(D \subset \widetilde{I})$, where $f_{1}\left(\mu_{1}\right), f_{2}\left(\mu_{2}\right), g_{1}\left(\nu_{1}\right)$, and $g_{2}\left(\nu_{2}\right)$ are monotonically increasing functions, $\tilde{A}$ is an IVIFN, and $\widetilde{X}_{0}$ is an accumulation point of $D$. If $\widetilde{X} \in S^{\oplus}\left(\widetilde{X}_{0}, F\right) \cap D$, then $\mid(F(\tilde{X}) \ominus$ $\left.F\left(\widetilde{X}_{0}\right)\right) \ominus\left(\widetilde{A} \otimes\left(\widetilde{X} \ominus \widetilde{X}_{0}\right)\right) \mid=o\left(\widetilde{X} \ominus \widetilde{X}_{0}\right)$; in this case, $F(\widetilde{X})$ is said to be addition differentiable at $\widetilde{X}_{0}$; if $\mid\left(F\left(\widetilde{X}_{0}\right) \ominus\right.$ $F(\widetilde{X})) \ominus\left(\widetilde{A} \otimes\left(\widetilde{X}_{0} \ominus \widetilde{X}\right)\right) \mid=o\left(\widetilde{X}_{0} \ominus \widetilde{X}\right)$ holds for $\widetilde{X} \in S^{\ominus}$ $\left(\widetilde{X}_{0}, F\right) \cap D$, then $F(\widetilde{X})$ is said to be subtraction differentiable at $\widetilde{X}_{0}$; to sum up, $F(\widetilde{X})$ is differentiable at $\widetilde{X}_{0}$, if $\|\left|F(\widetilde{X}) \ominus F\left(\widetilde{X}_{0}\right) \ominus\left(\widetilde{A} \otimes\left|\widetilde{X} \ominus \widetilde{X}_{0}\right|\right)=o\left(\left|\widetilde{X} \ominus \widetilde{X}_{0}\right|\right)\right|$ holds for $\widetilde{X} \in S^{\oplus}\left(\widetilde{X}_{0}, F\right) \cup S^{\ominus}\left(\widetilde{X}_{0}, F\right) \cap D$.

In what follows, we give the concrete value of $\tilde{A}$ in Definition 14:

Theorem 20. If $F(\widetilde{X})=\left(\left[f_{1}\left(\mu_{1}\right), f_{2}\left(\mu_{2}\right)\right],\left[g_{1}\left(v_{1}\right)\right.\right.$, $\left.\left.g_{2}\left(\nu_{2}\right)\right]\right)$ is differentiable at $\widetilde{X}_{0}$, and its domain is $D$, and if $\left|F(\widetilde{X}) \ominus F\left(\widetilde{X}_{0}\right) \ominus\left(\widetilde{A} \otimes\left|\widetilde{X} \ominus \widetilde{X}_{0}\right|||\right)=o\left(\left|\widetilde{X} \ominus \widetilde{X}_{0}\right|\right)\right|$ for $\tilde{X} \epsilon$ $\left(S^{\oplus}\left(\widetilde{X}_{0}, F\right) \cup S^{\ominus}\left(\widetilde{X}_{0}, F\right)\right) \cap D$, then $\widetilde{A}=\left(\left[\left(1-\mu_{10} / 1-f_{1}\right.\right.\right.$ $\left.\left.\left(\mu_{10}\right)\right) f_{1}^{\prime}\left(\mu_{10}\right),\left(1-\mu_{20} / 1-f_{2}\left(\mu_{20}\right)\right) f_{2}^{\prime}\left(\mu_{20}\right)\right], \quad\left[1-\left(v_{10} / g_{1}\right.\right.$ $\left.\left.\left.\left(\nu_{10}\right)\right) g_{1}^{\prime}\left(v_{10}\right), 1-\left(\nu_{20} / g_{2}\left(\nu_{20}\right)\right) g_{2}^{\prime}\left(v_{20}\right)\right]\right)$, where $\widetilde{A}$ is called the derivative of $F(\tilde{X})$ at $\widetilde{X}_{0}$, and denote it as $F^{\prime}\left(\widetilde{X}_{0}\right)$.

Proof. Without loss of generality, we only discuss $\widetilde{X} \in S^{\oplus}\left(\widetilde{X}_{0}, F\right) \cap D$; in this case, we have |([ $f_{1}\left(\mu_{1}\right), f_{2}$ $\left.\left(\mu_{2}\right)\right],\left[g_{1}\left(v_{1}\right), g_{2}\left(v_{2}\right)\right] \ominus\left[f_{1}\left(\mu_{10}\right), f_{2}\left(\mu_{20}\right)\right],\left[g_{1}\left(v_{10}\right), g_{2}\right.$ $\left.\left.\left(v_{20}\right)\right]\right) \ominus\left(\widetilde{A} \otimes\left(\left(\left[\mu_{1}, \mu_{2}\right],\left[v_{1}, v_{2}\right]\right) \ominus\left(\left[\mu_{10}, \mu_{20}\right],\left[v_{10}, v_{20}\right]\right)\right)\right) \mid$ $=o\left(\left(\left[\mu_{1}, \mu_{2}\right],\left[\nu_{1}, \nu_{2}\right]\right) \ominus\left(\left[\mu_{10}, \mu_{20}\right],\left[\nu_{10}, v_{20}\right]\right)\right)$.

As we know, the operation results of two IVIFNs consist of four parts: $\left(\left[\bullet_{1}, \bullet_{2}\right],\left[\bullet_{3}, \bullet_{4}\right]\right)$. First, we point out a fact that for the above formula, if we replace $\left(\left[\mu_{1}\right.\right.$, $\left.\left.\mu_{2}\right],\left[\nu_{1}, v_{2}\right]\right) \longrightarrow\left(\left[\mu_{10}, \mu_{20}\right],\left[v_{10}, v_{20}\right]\right)$ by $\left(\left[\mu_{1}, \mu_{1}\right]\right.$, $\left.\left[v_{1}, v_{1}\right]\right) \longrightarrow\left(\left[\mu_{10}, \mu_{10}\right],\left[v_{10}, v_{10}\right]\right)$, which does not affect the results of $\bullet_{1}$ and $\bullet_{3}$ for the above formula. In this case, the above formula is the differential of the IFF rather than that of the IVIFF, and thus, we can apply Theorem 3 to the above formula and obtain that $\bullet_{1}$ and $\bullet_{3}$ are $\left(1-\mu_{10} / 1-\right.$ $\left.f_{1}\left(\mu_{10}\right)\right) f_{1}^{\prime}\left(\mu_{10}\right)$ and $1-\left(\nu_{10} / g_{1}\left(\nu_{10}\right)\right) g_{1}^{\prime}\left(\nu_{10}\right)$, respectively. In the same way, we can obtain that $\bullet_{2}$ and $\bullet_{4}$ are $\left(1-\mu_{20} / 1-\right.$ $\left.f_{2}\left(\mu_{20}\right)\right) f_{2}^{\prime}\left(\mu_{20}\right)$ and $1-\left(v_{20} / g_{2}\left(v_{20}\right)\right) g_{2}^{\prime}\left(v_{20}\right)$, which completes the proof.

Moreover, the proof of Theorem 20 shows that $\widetilde{A}=$ $\lim _{\tilde{X} \longrightarrow \tilde{X}_{0}^{\oplus}}\left(F(\widetilde{X}) \ominus F\left(\widetilde{X}_{0}\right) / \widetilde{X} \ominus \widetilde{X}_{0}\right)$ for $\widetilde{X} \in S^{\oplus}\left(\widetilde{X}_{0}, F\right) \cap D$ and $\tilde{A}=\lim _{\widetilde{X} \longrightarrow \tilde{X}_{0}^{\ominus}}\left(F\left(\widetilde{X}_{0}\right) \ominus F(\widetilde{X}) / \widetilde{X}_{0} \ominus \widetilde{X}\right) \quad$ for $\quad \tilde{X} \in S^{\ominus}$ $\left(\widetilde{X}_{0}, F\right) \cap D$. When obtaining the value of $\widetilde{A}$, we only use the limit operation $\ominus$ and $\oslash$, and thus, according to Theorems 8 and $9, \widetilde{A}$ is also an IVIFN. Simultaneously, if recalling the proof of Theorem 1 [23], then we can omit the conditions $0 \leq\left(1-\mu_{20} / 1-f_{2}\left(\mu_{20}\right)\right) f_{2}^{\prime}\left(\mu_{20}\right) \leq\left(\nu_{20} / g_{2}\left(\nu_{20}\right)\right) g_{2}^{\prime}\left(\nu_{20}\right) \leq 1$, $0 \leq\left(1-\mu_{10} / 1-f_{1}\left(\mu_{10}\right)\right) f_{1}^{\prime}\left(\mu_{10}\right) \leq 1$, and $0 \leq \nu_{10} / g_{1}\left(\nu_{10}\right) g_{1}^{\prime}$ $\left(\nu_{10}\right) \leq 1$ since the interval-valued intuitionistic fuzzy metric space is complete.

Since the operational results of two IVIFNs consist of four parts: $\bullet_{1}, \bullet_{2}, \bullet_{3}$, and $\bullet_{4}$, and they are dependent each other. The following result can be obtained which is similar to the corresponding one in the IFC.

Theorem 21. $F(\tilde{X})$ is differentiable at a point $\widetilde{X}_{0}$ if and only if $F(\widetilde{X})$ has the derivative at $\tilde{X}_{0}$.

Lastly, for the addition and subtraction differential theory of IVIFFs, the relationship between the differential and the continuity is revealed as follows:

Theorem 22. If $F(\widetilde{X})$ is differentiable at $\widetilde{X}_{0} \in D$, then it is also continuous at $X_{0}$; if $F(\widetilde{X})$ is differentiable on a set $D$, then it is also continuous on the set $D$.

Proof. Without loss of generality, assume that $F(\tilde{X})$ is addition differentiable at $X_{0} \in E$, then $\lim _{\widetilde{X} \longrightarrow \widetilde{X}_{0}^{\oplus}}(F(X)$ $\left.\ominus F\left(\tilde{X}_{0}\right)\right)=\lim _{\tilde{X} \longrightarrow \tilde{X}_{0}^{\oplus}}\left(\left(F(\tilde{X}) \ominus F\left(\tilde{X}_{0}\right) / \tilde{X} \ominus \tilde{X}_{0}\right) \otimes\left(\tilde{X} \ominus \tilde{X}_{0}\right)\right)$ $=\lim _{\tilde{X} \longrightarrow \tilde{X}_{0}^{\oplus}}\left(F(\tilde{X}) \ominus F(\tilde{X}) / \tilde{X} \ominus \widetilde{X}_{0}\right) \otimes \lim _{\tilde{X} \longrightarrow \tilde{X}_{0}^{\oplus}}\left(\widetilde{X} \ominus \widetilde{X}_{0}\right)=$

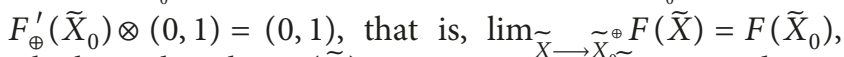
which implies that $F(\widetilde{X})$ is continuous at $\widetilde{X}_{0}$. Considering $X_{0} \in D$ and the arbitrariness of $X_{0}$, we can draw a conclusion that if $F(\widetilde{X})$ is differentiable on a set $D$, then it is also continuous on the set $D$, which completes the proof.

As far as the multiplication and the division differential theory of IVIFFs is concerned, we do not prepare to discuss them in detail; here, we only list a definition and a theorem:

Definition 15. An IVIFF $F\left(\widetilde{X}^{*}\right)=\left(\left[f_{1}\left(\mu_{1}\right), f_{2}\left(\mu_{2}\right)\right]\right.$, $\left.\left[g_{1}\left(v_{1}\right), g_{2}\left(v_{2}\right)\right]\right)$ is defined on a set $D(D \subset \widetilde{I})$, where $f_{1}\left(\mu_{1}\right), f_{2}\left(\mu_{2}\right), g_{1}\left(\nu_{1}\right)$, and $g_{2}\left(\nu_{2}\right)$ are monotonically increasing functions, $\widetilde{A}$ is an IVIFN, and $\widetilde{X}_{0}$ is an accumulation point of $D$. If $\widetilde{X}^{*} \in S^{\otimes}\left(\widetilde{X}_{0}, F\right) \cap D$, then $\left\langle\left(F\left(\widetilde{X}^{*}\right)\right.\right.$ $\left.\left.\oslash F\left(\widetilde{X}_{0}\right)\right) \oslash\left(\widetilde{A} \oplus\left(\tilde{X}^{*} \oslash \widetilde{X}_{0}\right)\right)\right\rangle=o\left(\tilde{X}^{*} \oslash \widetilde{X}_{0}\right)$; in this case, $F\left(\tilde{X}^{*}\right)$ is said to be multiplication differentiable at $\widetilde{X}_{0}$; if the equality $\left\langle\left(F\left(\widetilde{X}_{0}\right) \ominus F\left(\widetilde{X}^{*}\right)\right) \oslash\left(\widetilde{A} \oplus\left(\widetilde{X}_{0} \oslash \widetilde{X}^{*}\right)\right)\right\rangle=o\left(\widetilde{X}_{0} \oslash\right.$ $\left.\widetilde{X}^{*}\right)$ holds for $\widetilde{X} \in S^{\ominus}\left(\widetilde{X}_{0}, F\right) \cap D, F\left(\widetilde{X}^{*}\right)$ is said to be division differentiable at $\widetilde{X}_{0}$; to sum up, we say that $F\left(\widetilde{X}^{*}\right)$ is multiplication and division differentiable at $\widetilde{X}_{0}$, if $\langle\langle F$ $\left.\left.\left(\widetilde{X}^{*}\right) \oslash F\left(\widetilde{X}_{0}\right)\right\rangle \oslash\left(\widetilde{A} \oplus\left\langle\widetilde{X}^{*} \oslash \widetilde{X}_{0}\right\rangle\right)\right\rangle=o\left(\left\langle\widetilde{X}^{*} \oslash \widetilde{X}_{0}\right\rangle\right)$ holds for $\widetilde{X} \in S^{\oplus}\left(\widetilde{X}_{0}, F\right) \cup S^{\ominus}\left(\widetilde{X}_{0}, F\right) \cap D$.

Theorem 23. The following results are valid:

(a) If $F\left(\widetilde{X}^{*}\right)=\left(\left[f_{1}\left(\mu_{1}\right), f_{2}\left(\mu_{2}\right)\right],\left[g_{1}\left(\nu_{1}\right), g_{2}\left(\nu_{2}\right)\right]\right)$ is defined on a set $D(D \subset \widetilde{I})$ and multiplication and 
division differentiable at $\widetilde{X}_{0}$, and if $\left\langle\left\langle F\left(\widetilde{X}^{*}\right)\right.\right.$ $\left.\left.\oslash F\left(\widetilde{X}_{0}\right)\right\rangle \oslash\left(\widetilde{A} \oplus\left\langle\widetilde{X}^{*} \oslash \widetilde{X}_{0}\right\rangle\right)\right\rangle=o\left(\left\langle\widetilde{X}^{*} \oslash \widetilde{X}_{0}\right\rangle\right)$ for $\tilde{X} \in S^{\otimes}\left(\widetilde{X}_{0}, F\right) \cup S^{\ominus}\left(\widetilde{X}_{0}, F\right) \cap D$, then $\widetilde{A}=\left(\left[1-\left(\mu_{10}\right)\right.\right.$ $\left.\left.f_{1}\left(\mu_{10}\right)\right) f_{1}^{\prime}\left(\mu_{10}\right), 1-\left(\mu_{20} / f_{2}\left(\mu_{20}\right)\right) f_{2}^{\prime}\left(\mu_{20}\right)\right],\left[\left(1-v_{10} /\right.\right.$ $\left.\left.\left.1-g_{1}\left(\nu_{10}\right)\right) g_{1}^{\prime}\left(\nu_{10}\right),\left(1-\nu_{20} / 1-g_{2}\left(\nu_{20}\right)\right) g_{2}^{\prime}\left(\nu_{20}\right)\right]\right)$, where $\widetilde{A}$ is called the multiplication and division derivative of $F\left(\widetilde{X}^{*}\right)$ at $\tilde{X}_{0}$, and denote it as $F_{*}^{\prime}\left(\widetilde{X}_{0}\right)$.

(b) $F\left(\widetilde{X}^{*}\right)$ is multiplication and division differentiable at the point $\widetilde{X}_{0}$ if and only if $F\left(\widetilde{X}^{*}\right)$ has the multiplication and division derivative at $\widetilde{X}_{0}$.

(c) If $F\left(\tilde{X}^{*}\right)$ is multiplication and division differentiable at $\widetilde{X}_{0} \in D$, then it is also multiplication and division continuous at $X_{0}$; if $F\left(\widetilde{X}^{*}\right)$ is multiplication and division differentiable on a set $D$, then it is also multiplication and division continuous on the set D.

\section{Homomorphism between the IVIFNs and the IFNS}

As aforementioned, the IFC is based on the basic operational laws of IFNs, and correspondingly, the IVIFC is based on the basic operational laws of IVIFNs, and thus, it is necessary to reveal the relationships between the IFNs and the IVIFNs in this section.

As we know, for the IFNs and the IVIFNs, there are six basic operational laws: addition, subtraction, multiplication, division, scalar-multiplication, and power. In order to distinguish them, we denote the six basic operational laws as $\circ$

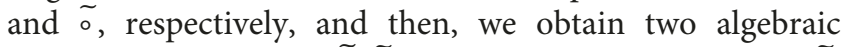
structures: $(I, \circ)$ and $(\widetilde{I}, \widetilde{\circ})$. If $\widetilde{\alpha}=\left(\left[\mu_{1}, \mu_{2}\right],\left[\nu_{1}, \nu_{2}\right]\right) \in \widetilde{I}$, we construct the map $\varphi\left(\left[\mu_{1}, \mu_{2}\right],\left[\nu_{1}, \nu_{2}\right]\right)=\left(\mu_{2}, \nu_{2}\right)$, obviously, the map is a surjection. However, it is not one-toone. For instance, $\varphi([0.2,0.4],[0.3,0.5])=(0.4,0.5)$ and $\varphi([0.1,0.4],[0.2,0.5])=(0.4,0.5)$. It follows from the basic operational laws for the IFNs and IVIFNs that $\varphi\left(\widetilde{\alpha}_{1} \circ \widetilde{\alpha}_{2}\right)=\varphi\left(\widetilde{\alpha}_{1}\right) \circ \varphi\left(\widetilde{\alpha}_{2}\right)$. For example, for the addition, $\varphi$ $\left(\widetilde{\alpha}_{1} \oplus \widetilde{\alpha}_{2}\right)=\varphi\left(\left[1-\left(1-\mu_{11}\right)\left(1-\mu_{12}\right), 1-\left(1-\mu_{21}\right)\left(1-\mu_{22}\right)\right]\right.$, $\left.\left[\nu_{11} \nu_{12}, v_{21} v_{22}\right]\right)=\left(1-\left(1-\mu_{21}\right) \quad\left(1-\mu_{22}\right), v_{21} v_{22}\right)$. Since $\varphi\left(\widetilde{\alpha}_{1}\right)=\left(\mu_{21}, \nu_{21}\right)$ and $\varphi\left(\widetilde{\alpha}_{2}\right)=\left(\mu_{22}, \nu_{22}\right)$, then $\varphi\left(\widetilde{\alpha}_{1}\right) \oplus \varphi\left(\widetilde{\alpha}_{2}\right)=\left(1-\left(1-\mu_{21}\right) \quad\left(1-\mu_{22}\right), \nu_{21} \nu_{22}\right)$, that is, $\varphi\left(\widetilde{\alpha}_{1} \oplus \widetilde{\alpha}_{2}\right)=\varphi\left(\widetilde{\alpha}_{1}\right) \oplus \varphi\left(\widetilde{\alpha}_{2}\right)$.

To sum up, in view of Definition 5 , we can verify that $(\widetilde{I}, \widetilde{\circ}) \sim(I, \circ)$. However, we cannot obtain $(I, \circ) \cong(\widetilde{I}, \widetilde{\circ})$, which means that $(I, \circ)$ and $(\widetilde{I}, \widetilde{\circ})$ does not have the same algebraic structure. From this point of view, it is necessary to discuss the IVIFC. Simultaneously, it should be noted that if $(\widetilde{I}, \widetilde{\circ}) \sim(I, \circ)$, then the two algebraic structures have some inner relationships: if $(\widetilde{I}, \widetilde{\circ})$ satisfies some operational laws, then $(I, \circ)$ meets the corresponding operational laws. In other words, if we develop the theory of the IVIFC, then we also enhance the theory of the IFC if we note that the IFC and the IVIFC are based on their basic operational laws, respectively, which is also in accordance with the fact that the IFN is a particular form of the IVIFN.

It is well known that if $(A, \widetilde{\circ}) \sim(B, \circ)$, then the homomorphism $\varphi$ can map the identity to the identity, and if $a_{1}, a_{2}, \cdots, a_{k}$ are the elements of $A$, then $\varphi\left(a_{1}{ }^{\circ}\right.$ $\left.a_{2} \sim \ldots \sim a_{k}\right)=\varphi\left(a_{1}\right) \circ \varphi\left(a_{2}\right) \circ \cdots \circ \varphi\left(a_{k}\right)$. In what follows, we only verify the former in intuitionistic fuzzy circumstance. $(\widetilde{I}, \oplus) \sim(I, \oplus)$, and the addition identity in $\widetilde{I}$ is $([0,0],[1,1]), \varphi(([0,0],[1,1]))=(0,1)$; it is clear that the addition identity in $I$ is $(0,1) ;(\widetilde{I}, \otimes) \sim(I, \otimes)$ and the multiplication identity in $\widetilde{I}$ is $([1,1],[0,0]), \varphi(([1,1]$, $[0,0]))=(1,0)$; it is obvious that the multiplication identity in $I$ is $(1,0)$. However, when we know that the addition identity in $I$ is $(0,1)$, we cannot deduce that the addition identity in $\widetilde{I}$ is $([0,0],[1,1])$ by the homomorphism $\varphi$ since $\varphi(([0,0],[1,1]))=(0,1)$ and $\varphi(([\underset{\sim}{0}, \underset{\sim}{0})$, $[0.7,1]))=(0,1)$, which also interpret that $(I, \circ)$ and $(\widetilde{I}, \widetilde{\circ})$ does not have the same algebraic structure.

We summarize this section via Figure 3. For the convenience of exhibition, we put $\varphi(\widetilde{\alpha})=\alpha$.

\section{Application to Continuous Data in Decision Making}

As for the IFC or the IVIFC, the authors have offered some applications in real life [19, 20, 22, 23]. Of course, following the idea in reference [23], we can easily give an application. However, in our opinion, it is not interesting and necessary. Particularly, to date, the researchers have not still offered the reason why we deal with continuous date in real life. And thus, the purpose of this section is to provide an application of continuous data in decision-making to show the necessity and feasibility of continuous data.

In the traditional decision-making, when the scholars handle the information of the IFNs and the IVIFNs, the data are usually discrete. However, in real life, some kinds of the data are continuous. For example, the power consumption of an electric appliance is continuous, which is a function of time; the displacement of a car is continuous and related to time; the height and weight of a person change with his/her ages, and so on. The IFMA and the IVIFMA focus on addressing continuous information of the IFNs and the IVIFNs, and as aforementioned, the scholars have successfully offered some applications in the previous literature $[19,20,22,23]$. For instance, Zhao et al. [23] offered the applications of the differentials of IVIFFSs in approximate calculations, and therefore, we do not prepare to provide similar applications although they can be easily obtained. In what follows, we offer an example to show that in some situations we have to use some techniques to address continuous information in the decision-making process since the data in nature are continuous, which can make the result be more reasonable than that by using the traditional techniques to address discrete data:

Example 7.1. A sales manager of a market wants to select one of two different brands of air conditioners which are of the same price. He invites two experts, $E_{1}$ and $E_{2}$, to evaluate their comfort, and he randomly selects an air conditioner for each brand; for convenience, we denote the two air conditioners as $A_{1}$ and $A_{2}$. The sales manager asks for $E_{1}$ to give his assessments to the comfort of $A_{1}$ every six minutes in an hour when $A_{1}$ operates in a closed house. Likewise, $E_{2}$ is responsible for evaluating the comfort of $A_{2}$ every six minutes in an hour 


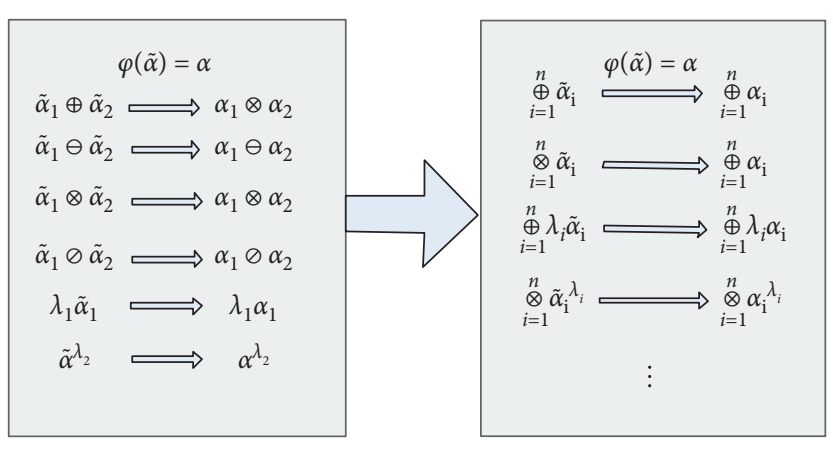

FIGURE 3: Interpretation of homomorphism.

when $A_{2}$ operates in another closed house. Since the criterion "comfort" is of vague nature, it is more suitable for $A_{1}$ and $A_{2}$ to express their assessments in the form of IVIFNs which can reflect the experts' hesitancy intuitively. Suppose that $E_{1}$ gives his assessments as $([0.08,0.11],[0.78,0.82]),([0.13$, $0.17],[0.68,0.79]),([0.26,0.34],[0.52,0.59]),([0.39,0.47]$, $[0.32,0.39]),([0.60,0.75],[0.22,0.24]), \quad([0.82,0.85],[0$. $10,0.11]),([0.72,0.78],[0.12,0.17]),([0.74,0.81],[0.06$, $0.13]),([0.65,0.70],[0.18,0.21])$, and $([0.68,0.73],[0.15$, $0.21])$; assume that $E_{2}$ gives his assessments as ([0.12, $0.15],[0.76,0.80]),([0.37,0.42],[0.45,0.51]),([0.60,0.62]$, $[0.26,0.33]),([0.78,0.83],[0.12,0.16]),([0.83,0.86],[0$. $06,0.08]), \quad([0.74,0.76],[0.10,0.13]),([0.71,0.72],[0.12$, $0.18]),([0.75,0.81],[0.10,0.13]),([0.81,0.85],[0.08,0.10])$, and $([0.73,0.75],[0.16,0.20])$. However, due to some objective reasons, the fourth assessment is obtained in the 27th minute rather than the 24th minute. Obviously, it is not suitable if we directly determine whose air conditioner is more comfortable in $A_{1}$ and $A_{2}$ only by the above data. How to get a reasonable result?

Indeed, the key issue is how to obtain the assessment of the 24th minute for $A_{2}$. Theoretically, due to the fact that there exist the assessments of "the comfort" every time if an air conditioner operates, and thus, for $A_{1}$ and $A_{2}$, all of the assessment $\mu_{1 n}, \mu_{2 n}, v_{1 n}$, and $v_{2 n}(n=1,2, \ldots, 10)$ are the continuous functions of the time $t$. It is well known that we apply the traditional techniques to handle discrete data. Since these above-mentioned data are continuous in essence, it is unreasonable to apply the traditional techniques to handle them. Now, we can obtain the assessment of the 24th minute for $A_{2}$ via data fitting. As shown in Figure 4, after normalizing the time $t$, we can get the function $\mu_{1}=f_{1}(t)$ by data fitting technique, and then, we have $f_{1}(0.4)=0.7696$. In the same way, we obtain that the assessment of the 24th minute is ([0.7696, 0.8072], [0.1268, 0.1648]); we take its approximate value $([0.77,0.81],[0.13,0.16])$ instead of the IVIFN $([0.78,0.83],[0.12,0.16])$.

TOPSIS (Technique for Order Preference by Similarity to Ideal Solution) is a famous method of decision-making. In what follows, using TOPSIS, we offer the result of Example 7.1 step by step:

Step 1. Identify the interval-valued intuitionistic fuzzy PIS (IVIF-PIS) $S_{i}^{+}$of each alternative $A_{i}$ and the intervalvalued intuitionistic fuzzy NIS (IVIF-NIS) $S_{i}^{-}$of each alternative $A_{i}$. We define the IVIF-PIS $S_{i}^{+}$and the IVIFNIS $S_{i}^{+}$as follows:

$$
\begin{aligned}
& S_{i}^{+}=\left(\left(\left[\max _{n}\left(\mu_{1 n}\right), \max _{n}\left(\mu_{2 n}\right)\right],\left[\min _{n}\left(v_{1 n}\right), \min _{n}\left(v_{2 n}\right)\right]\right)\right), \\
& S_{i}^{-}=\left(\left(\left[\min _{n}\left(\mu_{1 n}\right), \min _{n}\left(\mu_{2 n}\right)\right],\left[\max _{n}\left(v_{1 n}\right), \max _{n}\left(v_{2 n}\right)\right]\right)\right),
\end{aligned}
$$

and then,

$$
\begin{aligned}
& S_{1}^{+}=([0.82,0.85],[0.06,0.11]), \\
& S_{1}^{-}=([0.08,0.11],[0.78,0.82]), \\
& S_{2}^{+}=([0.83,0.86],[0.06,0.08]), \\
& S_{2}^{-}=([0.12,0.15],[0.76,0.80]) .
\end{aligned}
$$

Step 2. Calculate the separation measures $D_{i}^{+}$and $D_{i}^{-}$of each alternative $A_{i}$ from the $S_{i}^{+}$and $S_{i}^{-}$, respectively. We calculate them by the following formula:

$$
\begin{aligned}
& D_{i}^{+}=\sqrt{\frac{1}{20} \sum_{n=1}^{10}\left(\mu_{1 n}-\mu_{1 i}^{+}\right)^{2}+\left(\mu_{2 n}-\mu_{2 i}^{+}\right)^{2}+\left(\nu_{1 n}-\nu_{1 i}^{+}\right)^{2}+\left(\nu_{2 n}-v_{2 i}^{+}\right)^{2}}, \\
& D_{i}^{-}=\sqrt{\frac{1}{20} \sum_{n=1}^{10}\left(\mu_{1 n}-\mu_{1 i}^{-}\right)^{2}+\left(\mu_{2 n}-\mu_{2 i}^{-}\right)^{2}+\left(\nu_{1 n}-v_{1 i}^{-}\right)^{2}+\left(\nu_{2 n}-v_{2 i}^{-}\right)^{2}},
\end{aligned}
$$




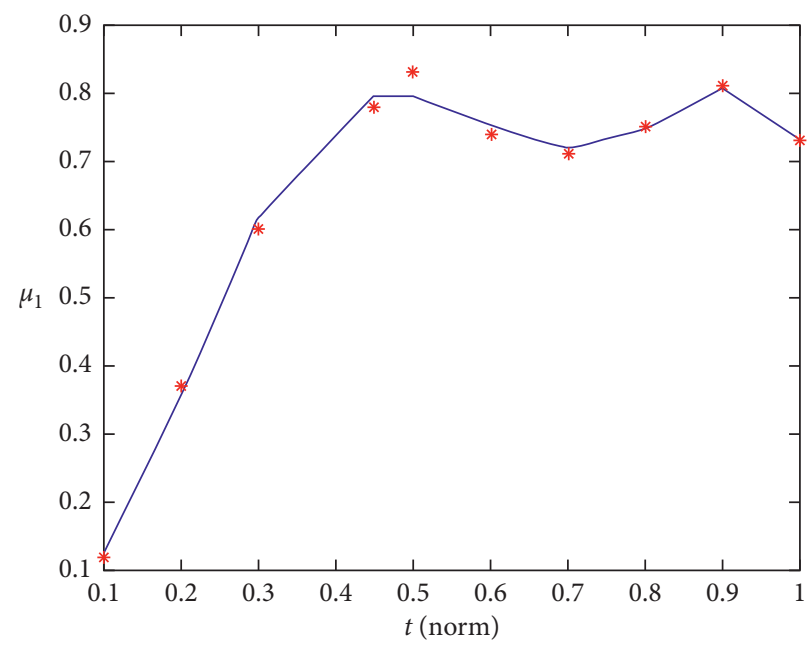

FIgURE 4: Data fitting of $\left(\mu_{1 i}, t\right)$ for $i=1,2, \cdots, 10$.

where $S_{i}^{+}=\left(\left[\mu_{1 i}^{+}, \mu_{2 i}^{+}\right],\left[\nu_{1 i}^{+}, v_{2 i}^{+}\right]\right)$and $S_{i}^{-}=\left(\left[\mu_{1 i}^{-}, \mu_{2 i}^{-}\right]\right.$, $\left.\left[v_{1 i}^{-}, v_{2 i}^{-}\right]\right)$. After a series of computations, we have $D_{1}^{+}=0.5317, D_{1}^{-}=0.7346, D_{2}^{+}=0.3839$, and $D_{2}^{-}=0.8492$.

Step 3. Find the closeness coefficient $C_{i}$ of each alternative $A_{i}$ by the formula $C_{i}=D_{i}^{-} / D_{i}^{-}+D_{i}^{+}$, and we get $C_{1}=0.5801$ and $C_{2}=0.6887$.

Step 4. Rank the alternatives and select the better alternatives in $A_{1}$ and $A_{2}$, the greater the value $C_{i}$, the better the alternative $A_{i}$. Since $C_{1}<C_{2}$, then the comfort of $A_{2}$ is better than that of $A_{1}$.

Finally, we offer a comparison of $\mu_{1}$ between $A_{1}$ and $A_{2}$. Figure 5 shows that the comfort of $A_{2}$ is superior to that of $A_{1} ; A_{2}$ can quickly reach the biggest assessment only in 25 minutes and $A_{1}$ reaches the biggest assessment in 30 minutes. Moreover, after they reach the biggest values, the temperature range of variation for $A_{2}$ is smaller than that of $A_{1}$. In short, if we further investigate the data, then we can see that our result is reasonable.

Let us apply the IFWA operators [27] IFWA $\left(\alpha_{1}\right.$, $\left.\alpha_{2}, \cdots, \alpha_{m}\right)=\oplus_{i=1}^{m} \omega_{i} \alpha_{i}=\left(1-\prod_{i=1}^{m}\left(1-\mu_{\alpha_{i}}\right)^{\omega_{i}}, \prod_{i=1}^{m} v_{\alpha_{i}}^{\omega_{i}}\right)$ to solve the above example; for each interval, if we take its midpoint and if the weights are equal, then we also have that the comfort of $A_{2}$ is better than that of $A_{1}$. However, the fourth assessment is obtained in the 27 th minute rather than the 24th minute. In other words, we cannot obtain the fourth accurate assessment via the IFWA operators. Even if we can obtain the fourth accurate assessment, we have to face the fact that the aggregation result via the IFWA operators is related to the weight of each IVIFN. However, the proposed method does not face this predicament. Indeed, sometimes it is difficult for the decision makers to give the weight of each assessment in real life. And thus, our proposed method is reasonable and progressive.

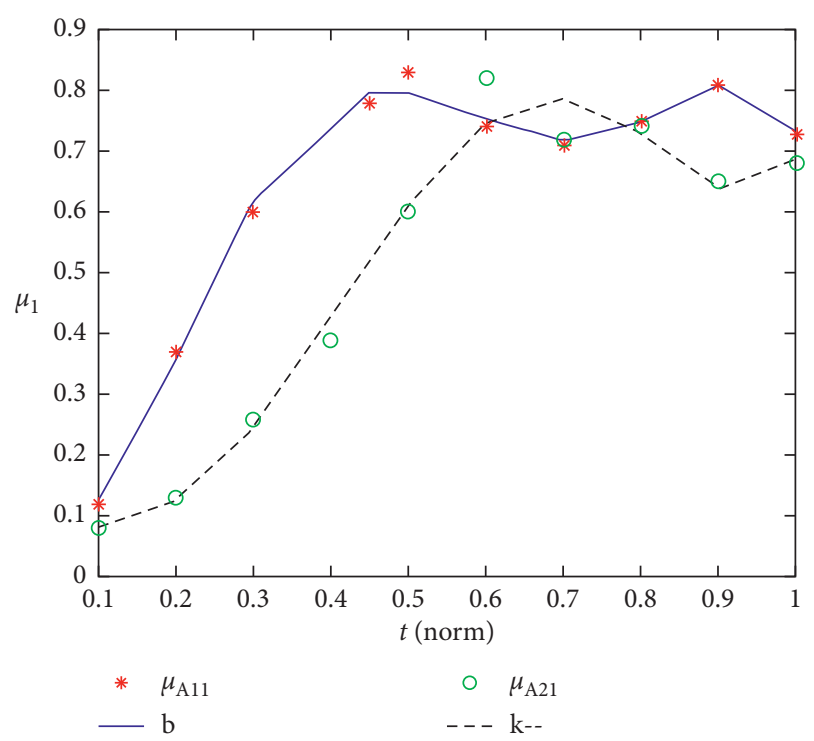

Figure 5: A comparison of $\mu_{1}$ between $A_{1}$ and $A_{2}$.

\section{Concluding Remarks}

In this paper, we have studied the limit theory of the intervalvalued intuitionistic fuzzy sequences, in order to avoid some tedious computations. By introducing the interval-valued intuitionistic fuzzy metric space, we have obtained a series of properties whose proofs are considerably concise, which also means that the intuitionistic fuzzy operational laws have many considerably good properties. In other words, if these operational laws were bad, we would have to face some tedious computations. We have also described the characterization of the limit, the continuity, and the differential with respect to the IVIFFs and revealed their relationships. In the literature [17], the authors did not insist on using the two symbols $|\bullet|$ and $\langle\bullet\rangle$ to discuss the limit, the continuity, 
and the differential theory of IFFs. In this paper, we have overcome the weakness, which can greatly simplify the process when we discuss the IVIFC. For the differential theory, following the traditional calculus, we have first offered the definitions of differentials which are based on the IVIFIs, and then, we have obtained the derivatives, which are thoroughly different from those of the existing literature. As mentioned in Introduction, the beauty of mathematics is not complicated computation but her brilliant thoughts. After offering the definitions of the differentials, we have sought out the inner relationships between the intuitionistic fuzzy differentials and the interval-valued intuitionistic fuzzy differentials, and we have easily obtained the concrete values of two constants in the definitions of the differentials. Subsequently, considering that both the IFC and the IVIFC are based on the basic operational laws of the IFNs and IVIFNs, respectively. We have revealed the relationships between the IFNs and the IVIFNs: they are homomorphic rather than isomorphic, which means that they are two different algebraic structures, and which makes the discussion of the IVIFC be necessary. Indeed, by homomorphic mappings, theoretically, we have also interpreted the reason why there exist abundant papers with respect to IVIFNs since the structure of IFNs are different that of IVIFNs. As usual, the scholars only offered the reasons why they discussed IVIFNs in decision-making from the perspectives of the human thought characteristic. Finally, we have offered an application to continuous data with respect to IVIFNs to reveal the fact that in real life we occasionally handle continuous data since they are continuous in essence, and the example has shown the advantages of continuous data in the decision-making processes. Particularly, compared to the IFWA operators, the proposed method does not depend on the weights of the assessments, which make the result be more objective. In future research, we will focus on the practical applications to IVIFC and give other forms to further reveal its advantages in dealing with continuous data. We will also combine the other generalization [28] of the FSs to investigate continuous data and provide its applications in more complicated circumstances.

\section{Data Availability}

No data were used to support this study.

\section{Conflicts of Interest}

The authors declare that they have no conflicts of interest.

\section{Acknowledgments}

This work was supported by the Research project of Leshan Normal University (No. 801419004).

\section{References}

[1] L. A. Zadeh, "Fuzzy sets," Information and Control, vol. 8, no. 3, pp. 338-353, 1965.

[2] K. Atanassov, Intuitionistic Fuzzy Sets, 7th Youth Scientific Session, ITCRBAS, Sofia, Bulgaria, 1983.
[3] V. Torra, "Hesitant fuzzy sets," International Journal of Intelligent Systems, vol. 25, pp. 529-539, 2010.

[4] J. Aisbett, J. Rickard, and D. Morgenthaler, "Type-2 fuzzy sets as functions on spaces," IEEE Transactions on Fuzzy Systems, vol. 18, no. 4, pp. 841-844, 2010.

[5] B. Zhu, Z. S. Xu, and M. M. Xia, "Dual hesitant fuzzy sets," Journal of Applied Mathematics, vol. 2012, Article ID 879629, 13 pages, 2012.

[6] R. M. Rodriguez, L. Martinez, and F. Herrera, "Hesitant fuzzy linguistic term sets for decision making," IEEE Transactions on Fuzzy Systems, vol. 20, no. 1, pp. 109-119, 2012.

[7] K. Atanassov and G. Gargov, "Interval valued intuitionistic fuzzy sets," Fuzzy Sets and Systems, vol. 31, no. 3, pp. 343-349, 1989.

[8] P. Liu, "Some geometric aggregation operators based on interval intuitionistic uncertain linguistic variables and their application to group decision making," Applied Mathematical Modelling, vol. 37, no. 4, pp. 2430-2444, 2013.

[9] W. Wang, X. Liu, and Y. Qin, "Interval-valued intuitionistic fuzzy aggregation operators," Journal of Systems Engineering and Electronics, vol. 23, no. 4, pp. 574-580, 2012.

[10] Z. Xu, "A method based on distance measure for intervalvalued intuitionistic fuzzy group decision making," Information Sciences, vol. 180, no. 1, pp. 181-190, 2010.

[11] L. D. Miguel, H. Bustince, J. Fernandez, and R. Mesiar, "Construction of admissible linear orders for interval-valued Atanassov intuitionistic fuzzy sets with an application to decision making," Information Fusion, vol. 27, pp. 189-197, 2016.

[12] S.-p. Wan, G.-l. Xu, and J.-y. Dong, "A novel method for group decision making with interval-valued Atanassov intuitionistic fuzzy preference relations," Information Sciences, vol. 372, pp. 53-71, 2016.

[13] J. Wu and F. Chiclana, "Non-dominance and attitudinal prioritisation methods for intuitionistic and interval-valued intuitionistic fuzzy preference relations," Expert Systems with Applications, vol. 39, no. 18, pp. 13409-13416, 2012.

[14] C.-P. Wei, P. Wang, and Y.-Z. Zhang, "Entropy, similarity measure of interval-valued intuitionistic fuzzy sets and their applications," Information Sciences, vol. 181, no. 19, pp. 4273-4286, 2011.

[15] J. Ye, "Fuzzy cross entropy of interval-valued intuitionistic fuzzy sets and its optimal decision-making method based on the weights of alternatives," Expert Systems with Applications, vol. 38, no. 5, pp. 6179-6183, 2011.

[16] Q. Lei and Z. Xu, "Derivative and differential operations of intuitionistic fuzzy numbers," International Journal of Intelligent Systems, vol. 30, no. 4, pp. 468-498, 2015.

[17] Z. Ai, Z. Xu, and Q. Lei, "Limit properties and derivative operations in the metric space of intuitionistic fuzzy numbers," Fuzzy Optimization and Decision Making, vol. 16, no. 1, pp. 71-87, 2017.

[18] Z. Ai, Z. Xu, and Q. Lei, "Fundamental properties with respect to the completeness of intuitionistic fuzzy partially ordered set," IEEE Transactions on Fuzzy Systems, vol. 25, no. 6, pp. 1741-1751, 2017.

[19] Q. Lei and Z. Xu, "Fundamental properties of intuitionistic fuzzy calculus," Knowledge-based Systems, vol. 76, pp. 1-16, 2015.

[20] Q. Lei and Z. Xu, "Relationships between two types of intuitionistic fuzzy definite integrals," IEEE Transactions on Fuzzy Systems, vol. 24, no. 6, pp. 1410-1425, 2016.

[21] Z. Ai and Z. Xu, "Multiple definite integrals of intuitionistic fuzzy calculus and isomorphic mappings," IEEE Transactions on Fuzzy Systems, vol. 26, no. 2, pp. 670-680, 2018. 
[22] Z. Ai and Z. Xu, "Line integrals of intuitionistic fuzzy calculus and their properties," IEEE Transactions on Fuzzy Systems, vol. 26, no. 3, pp. 1435-1446, 2018.

[23] H. Zhao, Z. Xu, and Z. Yao, "Interval-valued intuitionistic fuzzy derivative and differential operations," International Journal of Computational Intelligence Systems, vol. 9, no. 1, pp. 36-56, 2016.

[24] M. Kline, Mathematical Thought from Ancient to Modern Times, Vol. 2, Oxford University Press, New York, NY, USA, 1972.

[25] Z. S. Xu, "Methods for aggregation interval-valued intuitionistic fuzzy information and their application to decision making," Control and Decision, vol. 22, pp. 215-219, 2007.

[26] J. B. Fraleigh, A First Course in Abstract Algebra, AddisonWesley, New Jersey, NY, USA, 7th edition, 2002.

[27] Z. S. Xu, "Intuitionistic fuzzy aggregation operations," IEEE Transactions on Fuzzy Systems, vol. 15, no. 6, pp. 1179-1187, 2007.

[28] X. D. Peng and G. Selvachandran, "Pythagorean fuzzy set: state of the art and future directions," Artificial Intelligence Review, vol. 52, pp. 1873-1927, 2017. 


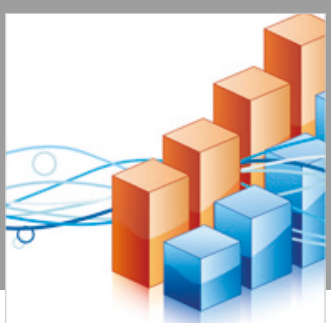

Advances in

Operations Research

\section{-n-m}
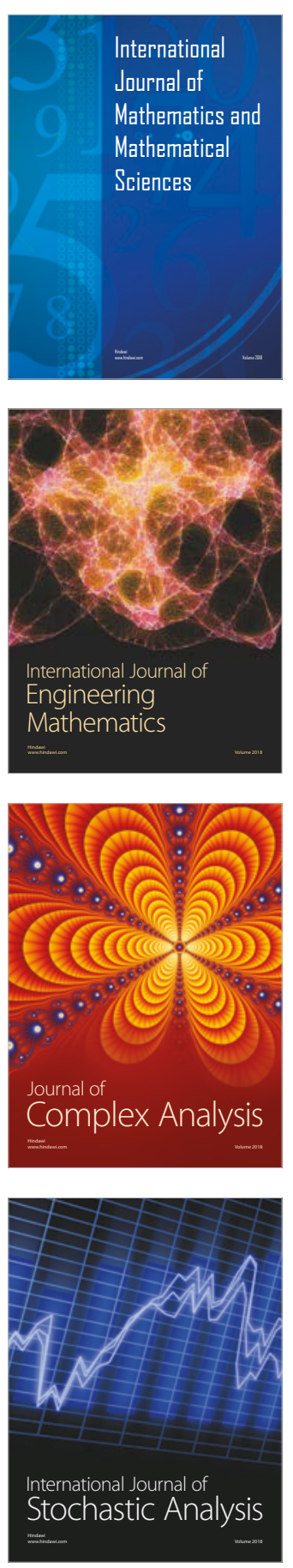
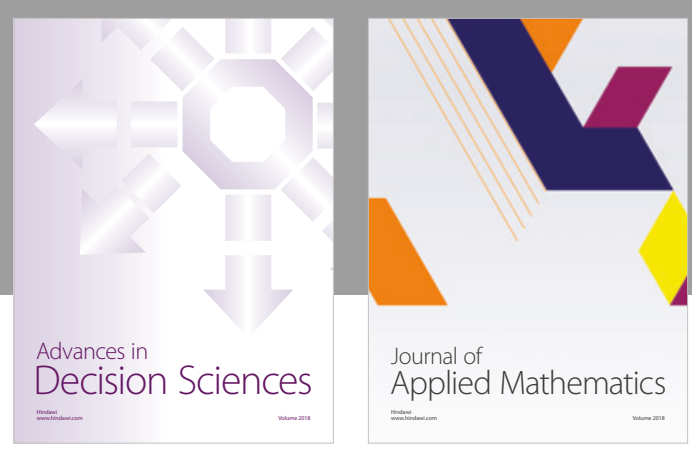

Journal of

Applied Mathematics
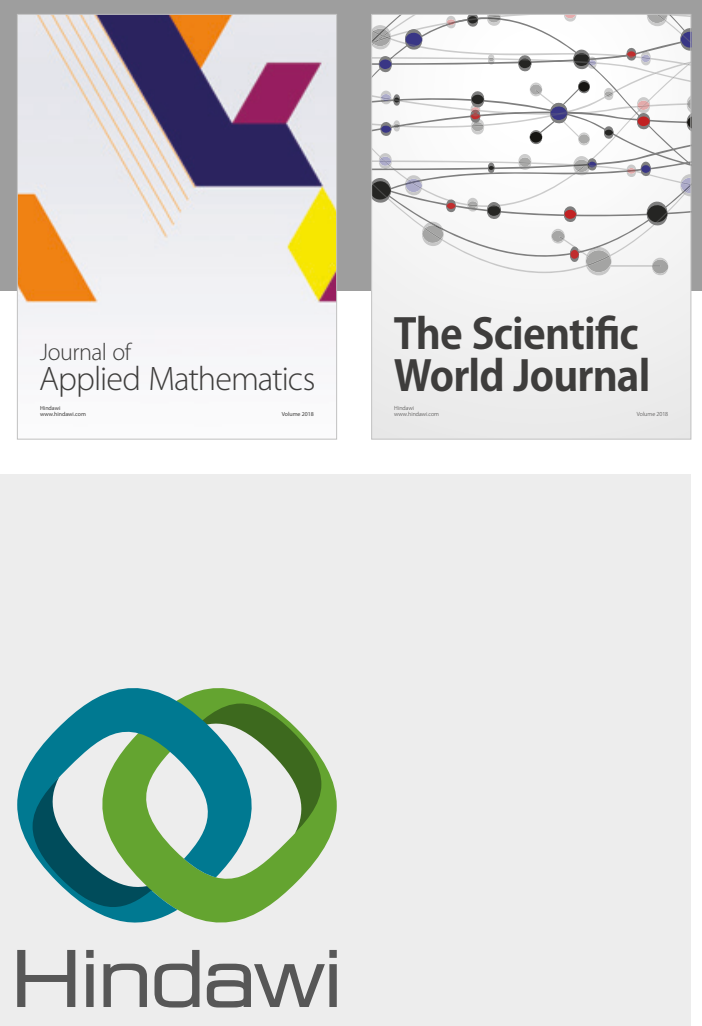

Submit your manuscripts at

www.hindawi.com

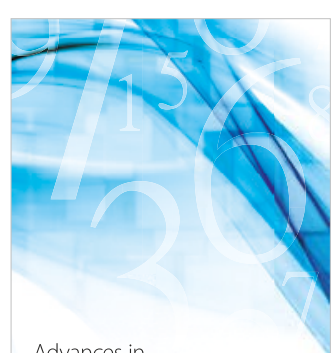

Advances in
Numerical Analysis
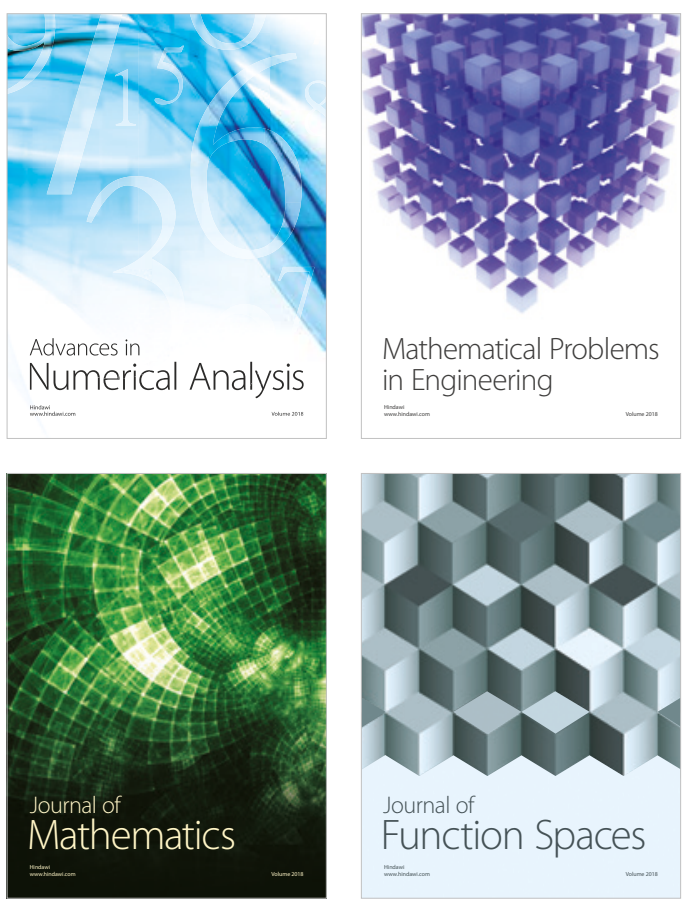

Mathematical Problems in Engineering

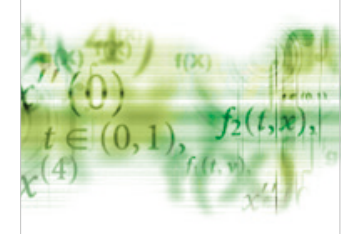

International Journal of

Differential Equations

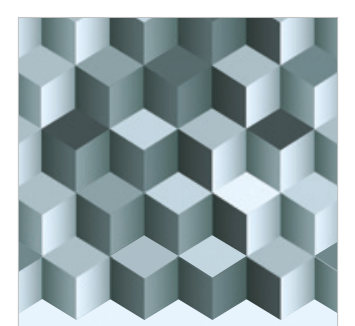

Journal of

Function Spaces
The Scientific

World Journal

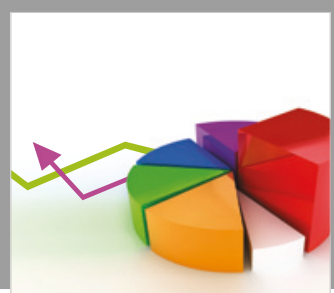

Journal of

Probability and Statistics
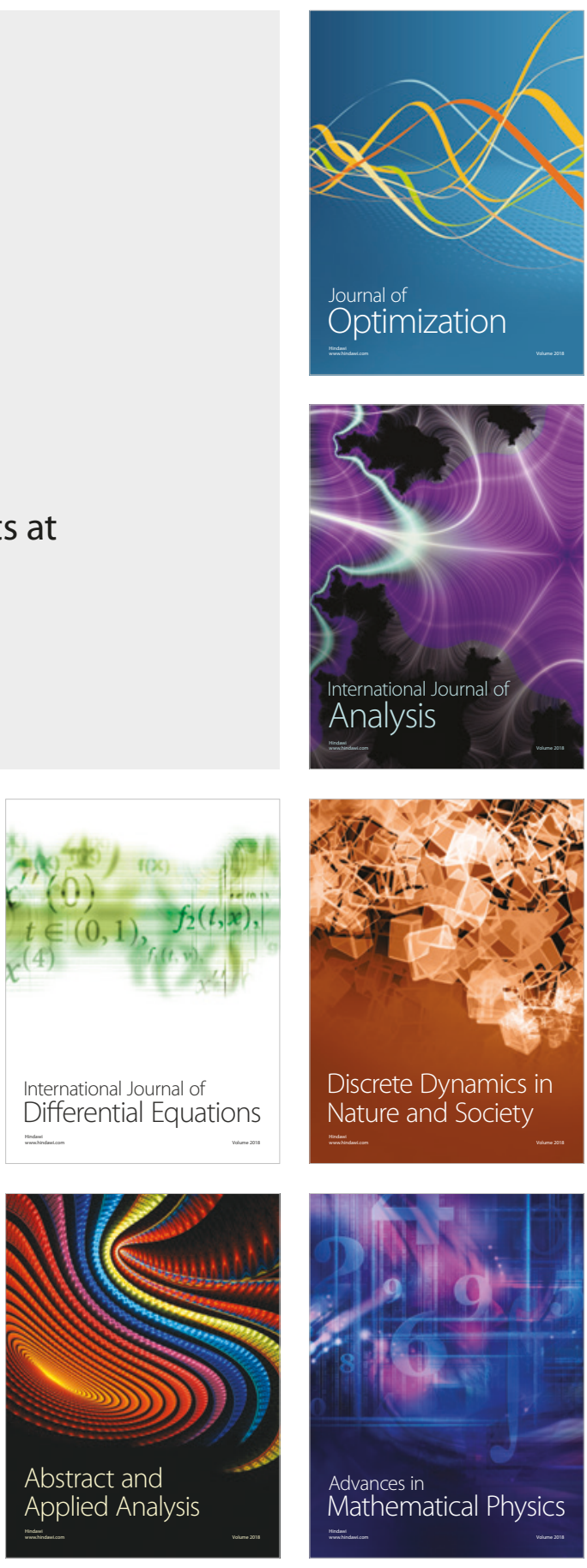